\title{
Local existence of strong solutions to the $k-\varepsilon$ model equations for turbulent flows
}

\author{
Baoquan Yuan* and Guoquan Qin
}

"Correspondence:

bqyuan@hpu.edu.cn

School of Mathematics and Information Science, Henan

Polytechnic University, Henan, 454000, China

\begin{abstract}
In this paper, we are concerned with the local existence of strong solutions to the $k-\varepsilon$ model equations for turbulent flows in a bounded domain $\Omega \subset \mathbb{R}^{3}$. We prove the existence of unique local strong solutions under the assumption that the turbulent kinetic energy and the initial density both have lower bounds away from zero.
\end{abstract}

MSC: 35Q35; 76F60; 76N10

Keywords: $k-\varepsilon$ model equations; strong solution; local well-posedness

\section{Introduction}

Turbulence is a natural phenomenon, which occurs inevitably when the Reynolds number of flows becomes high enough $\left(10^{6}\right.$ or more). In this paper, we consider the $k-\varepsilon$ model equations $[1,2]$ for turbulent flows in a bounded domain $\Omega \subset \mathbb{R}^{3}$ with smooth boundary,

$$
\begin{aligned}
& \rho_{t}+\nabla \cdot(\rho u)=0, \\
& (\rho u)_{t}+\nabla \cdot(\rho u \otimes u)-\Delta u-\nabla(\nabla \cdot u)+\nabla p=-\frac{2}{3} \nabla(\rho k), \\
& (\rho h)_{t}+\nabla \cdot(\rho u h)-\Delta h=p_{t}+u \cdot \nabla p+S_{k}, \\
& (\rho k)_{t}+\nabla \cdot(\rho u k)-\Delta k=G-\rho \varepsilon, \\
& (\rho \varepsilon)_{t}+\nabla \cdot(\rho u \varepsilon)-\Delta \varepsilon=\frac{C_{1} G \varepsilon}{k}-\frac{C_{2} \rho \varepsilon^{2}}{k}, \\
& (\rho, u, h, k, \varepsilon)(x, 0)=\left(\rho_{0}(x), u_{0}(x), h_{0}(x), k_{0}(x), \varepsilon_{0}(x)\right), \\
& \left.\left(u \cdot \vec{n}, h, \frac{\partial k}{\partial \vec{n}}, \frac{\partial \varepsilon}{\partial \vec{n}}\right)\right|_{\partial \Omega}=(0,0,0,0),
\end{aligned}
$$

with

$$
\begin{aligned}
& S_{k}=\left[\mu\left(\frac{\partial u^{i}}{\partial x_{j}}+\frac{\partial u^{j}}{\partial x_{i}}\right)-\frac{2}{3} \delta_{i j} \frac{\partial u^{k}}{\partial x_{k}}\right] \frac{\partial u^{i}}{\partial x_{j}}+\frac{\mu_{t}}{\rho^{2}} \frac{\partial p}{\partial x_{j}} \frac{\partial \rho}{\partial x_{j}}, \\
& G=\frac{\partial u^{i}}{\partial x_{j}}\left[\mu_{e}\left(\frac{\partial u^{i}}{\partial x_{j}}+\frac{\partial u^{j}}{\partial x_{i}}\right)-\frac{2}{3} \delta_{i j}\left(\rho k+\mu_{e} \frac{\partial u^{k}}{\partial x_{k}}\right)\right], \\
& p=\rho^{\gamma}
\end{aligned}
$$

(0) 2016 Yuan and Qin. This article is distributed under the terms of the Creative Commons Attribution 4.0 International License (http://creativecommons.org/licenses/by/4.0/), which permits unrestricted use, distribution, and reproduction in any medium, provided you give appropriate credit to the original author(s) and the source, provide a link to the Creative Commons license, and indicate if changes were made. 
where $\delta_{i j}=0$ if $i \neq j, \delta_{i j}=1$ if $i=j$, and $\mu, \mu_{t}, \mu_{e}, C_{1}$, and $C_{2}$ are five positive constants satisfying $\mu+\mu_{t}=\mu_{e}$, and $\vec{n}$ is the unit outward normal to $\partial \Omega$.

Equations (1.1)-(1.10) are derived from combining the effect of turbulence on the timeaveraged Navier-Stokes equations with the $k-\varepsilon$ model equations. The unknown functions $\rho, u, h, k$, and $\varepsilon$ denote the density, velocity, total enthalpy, turbulent kinetic energy, and the rate of viscous dissipation of turbulent flows, respectively. The expression of the pressure $p$ has been simplified here, which indeed has no bad effect on our study.

In partial differential equations, the $k-\varepsilon$ equations belong to the compressible ones. In this regard, we will refer to the classical compressible Navier-Stokes equations and compressible MHD equations, which are also research mainstreams, to carry out our study.

For compressible isentropic Navier-Stokes equations, the first question provoking our interest is the existence of the weak solutions. Lions $[3,4]$ proved the global existence of weak solutions under the condition that $\gamma>\frac{3 n}{n+2}$, where $\gamma$ is the same as in (1.10) and $n$ is the dimension of space. Later, Feireisl $[5,6]$ improved his result to $\gamma>\frac{n}{2}$. The condition satisfied by $\gamma$ is to prove the existence of renormalized solutions, which were introduced by DiPerna and Lions [7]. When the initial data are general small perturbations of non-vacuum resting state, Hoff [8] proved the global existence of weak solutions provided $\gamma>1$. The existence of strong solutions is another problem provoking our interest in the research of Navier-Stokes equations. It has been proved that the density will be away from vacuum at least in a small time interval provided the initial density is positive. If the initial data have better regularity, the compressible isentropic Navier-Stokes equations will admit a unique local strong solution under various boundary conditions [9-12]. However, when the initial vacuum is allowed, it was shown recently in [9] that the isentropic one will have a local strong solution in the case that some compatibility conditions are satisfied initially. Choe and Kim [13] obtained the unique local strong solutions for full compressible polytropic Navier-Stokes equations under a similar condition in [9]. In [13], the technique the authors used is mainly the standard iteration argument and the key point of their success is the estimate for the $L^{2}$ norm of the gradient of the pressure. In the process of studying the condition of a local solution becoming a global one, Xin [14] proved that the smooth solutions will blow up in finite time when an initial vacuum is allowed.

As for compressible MHD equations, the research directions, which mainly contain first the existence of weak and strong solutions and second the condition of weak solutions becoming a strong or even classical one and the local becoming a global one, are similar to that of Navier-Stokes equations. For example, Hu and Wang [15-17] obtained the local existence of weak solutions to the compressible isentropic MHD equations. Rozanova [18] proved the local existence of classical solutions to the compressible barotropic MHD equations provided both the mass and energy are finite. Fan and $\mathrm{Yu}$ in [19] proved the existence and uniqueness of strong solutions to the full compressible MHD equations. The method Fan and Yu [19] used is similar to that in [13], for example, they are both dependent on the standard iteration argument and the estimate for the $L^{2}$ norm of the gradient of the pressure.

In this paper, we consider the existence of strong solutions to the $k-\varepsilon$ model equations (1.1)-(1.10) in a bounded domain $\Omega \subset \mathbb{R}^{3}$. Our method is similar to that in [19] and [13]. However, in the process of applying the method to the $k-\varepsilon$ model equations, we find that the regularity of the solutions should be higher, which is induced by the higher nonlinearity in the compressible Navier-Stokes equations and compressible MHD equa- 
tions than that in [19] and [13]. In fact, when we make the difference of the $n$th and the $(n+1)$ th cases of equation (2.4) and integrating the result, we inevitably arrive at the term $\int \partial_{j} \bar{\rho}^{n+1} \partial_{j} \rho^{n+1} \cdot \bar{h}^{n+1}$. Therefore, we have to use integration by parts, which leads to two terms as $\int \bar{\rho}^{n+1} \partial_{j} \partial_{j} \rho^{n+1} \cdot \bar{h}^{n+1}$ and $\int \bar{\rho}^{n+1} \partial_{j} \rho^{n+1} \cdot \partial_{j} \bar{h}^{n+1}$. Then, by the Hölder and Young inequalities, it turns out that $\left\|\nabla^{2} \rho^{n+1}\right\|_{L^{3}}$ and $\left\|\nabla \rho^{n+1}\right\|_{L^{\infty}}$ should be bounded. Thus, we need $\|\rho\|_{H^{3}}$ to be bounded for an a priori estimate. Therefore, from the mass equation enough regularity of the velocity field should be imposed. Moreover, due to the strong-coupling property of the $k-\varepsilon$ equations, we need a corresponding high regularity of the unknown functions $k$ and $\varepsilon$.

Stated simply, the high nonlinearity of the $k-\varepsilon$ equations leads to the necessity of high regularity of some unknown functions and thus leads to much difficulties for the $a$ priori estimates. Besides, physically, when the turbulent kinetic energy $k$ vanish, the turbulence will disappear and the $k-\varepsilon$ model equations will degenerate into the Navier-Stokes equation. Therefore, without loss of generality, we assume throughout this paper that the turbulent kinetic energy $k$ has a positive lower bound away from zero, namely, $0<m<k$ with $m$ a constant.

To conclude this introduction, we give the outline of the rest of this paper: In Section 2, we consider a linearized problem of the $k-\varepsilon$ equations and derive some local-in-time estimates for the solutions of the linearized problem. In Section 3, we prove the existence theorem of the local strong solution of the original nonlinear problem.

\section{A priori estimates for a linearized problem}

Using the density equation (1.1), we could change (1.1)-(1.10) into the following equivalent form:

$$
\left\{\begin{array}{l}
\rho_{t}+\nabla \cdot(\rho u)=0, \\
\rho u_{t}+\rho u \cdot \nabla u-\Delta u-\nabla \operatorname{div} u+\nabla p=-\frac{2}{3} \nabla(\rho k), \\
\rho h_{t}+\rho u \cdot \nabla h-\Delta h=p_{t}+u \cdot \nabla p+S_{k}, \\
\rho k_{t}+\rho u \cdot \nabla k-\Delta k=G-\rho \varepsilon, \\
\rho \varepsilon_{t}+\rho u \cdot \nabla \varepsilon-\Delta \varepsilon=\frac{C_{1} G \varepsilon}{k}-\frac{C_{2} \rho \varepsilon^{2}}{k}, \\
(\rho, u, h, k, \varepsilon)(x, 0)=\left(\rho_{0}(x), u_{0}(x), h_{0}(x), k_{0}(x), \varepsilon_{0}(x)\right), \\
\left.\left(u \cdot \vec{n}, h, \frac{\partial k}{\partial \vec{n}}, \frac{\partial \varepsilon}{\partial \vec{n}}\right)\right|_{\partial \Omega}=(0,0,0,0) .
\end{array}\right.
$$

Then we consider the following linearized problem of (2.1):

$$
\begin{aligned}
& \rho_{t}+\nabla \cdot(\rho v)=0, \\
& \rho u_{t}+\rho v \cdot \nabla u-\Delta u-\nabla \operatorname{div} u+\nabla p=-\frac{2}{3} \nabla(\rho \pi), \\
& \rho h_{t}+\rho v \cdot \nabla h-\Delta h=p_{t}+u \cdot \nabla p+S_{k}^{\prime}, \\
& \rho k_{t}+\rho v \cdot \nabla k-\Delta k=G^{\prime}-\rho \theta, \\
& \rho \varepsilon_{t}+\rho v \cdot \nabla \varepsilon-\Delta \varepsilon=\frac{C_{1} G^{\prime} \theta}{\pi}-\frac{C_{2} \rho \theta^{2}}{\pi}, \\
& (\rho, v, h, \pi, \theta)(x, 0)=\left(\rho_{0}(x), u_{0}(x), h_{0}(x), k_{0}(x), \varepsilon_{0}(x)\right), \\
& \left.\left(v \cdot \vec{n}, h, \frac{\partial \pi}{\partial \vec{n}}, \frac{\partial \theta}{\partial \vec{n}}\right)\right|_{\partial \Omega}=(0,0,0,0),
\end{aligned}
$$


with

$$
\begin{aligned}
& S_{k}^{\prime}=\left[\mu\left(\frac{\partial v^{i}}{\partial x_{j}}+\frac{\partial v^{j}}{\partial x_{i}}\right)-\frac{2}{3} \delta_{i j} \frac{\partial v^{k}}{\partial x_{k}}\right] \frac{\partial v^{i}}{\partial x_{j}}+\frac{\mu_{t}}{\rho^{2}} \frac{\partial p}{\partial x_{j}} \frac{\partial \rho}{\partial x_{j}}, \\
& G^{\prime}=\frac{\partial v^{i}}{\partial x_{j}}\left[\mu_{e}\left(\frac{\partial v^{i}}{\partial x_{j}}+\frac{\partial v^{j}}{\partial x_{i}}\right)-\frac{2}{3} \delta_{i j}\left(\rho \pi+\mu_{e} \frac{\partial v^{k}}{\partial x_{k}}\right)\right],
\end{aligned}
$$

where $v, \pi$, and $\theta$ are known quantities on $\left(0, T_{1}\right) \times \Omega$ with $T_{1}>0$.

Here we also impose the following regularity conditions on the initial data:

$$
\left\{\begin{array}{l}
0<m<\rho_{0}, \quad \rho_{0} \in H^{3}(\Omega), \\
u_{0} \in H^{3}(\Omega), \\
\left(h_{0}, k_{0}, \varepsilon_{0}\right) \in H^{2}(\Omega), \\
\left.\left(u_{0} \cdot \vec{n}, h_{0}, \frac{\partial k_{0}}{\partial \vec{n}}, \frac{\partial \varepsilon_{0}}{\partial \vec{n}}\right)\right|_{\partial \Omega}=(0,0,0,0), \\
0<m<k_{0} .
\end{array}\right.
$$

For the known quantities $v, \pi, \theta$, we assume that $v(0)=u_{0}, \pi(0)=k_{0}, \theta(0)=\varepsilon_{0}$, and

$$
\left\{\begin{array}{l}
\sup _{0 \leq t \leq T_{2}}\left(\|v\|_{H^{1}}+\|\pi\|_{H^{1}}+\|\theta\|_{H^{1}}\right) \\
\quad+\int_{0}^{T_{2}}\left(\|\pi\|_{H^{3}}^{2}+\left\|v_{t}\right\|_{H^{1}}^{2}+\left\|\pi_{t}\right\|_{H^{1}}^{2}+\left\|\theta_{t}\right\|_{H^{1}}^{2}\right) \mathrm{d} t \leq c_{1}, \\
\sup _{0 \leq t \leq T_{2}}\|v\|_{H^{2}} \leq c_{2}, \\
\sup _{0 \leq t \leq T_{2}}\|v\|_{H^{3}} \leq c_{3}, \\
\int_{0}^{T_{2}}\|v\|_{H^{4}}^{2} \mathrm{~d} t \leq c_{4}, \\
\sup _{0 \leq t \leq T_{2}}\|\pi\|_{H^{2}} \leq c_{5}, \\
\sup _{0 \leq t \leq T_{2}}\|\theta\|_{H^{2}} \leq c_{6},
\end{array}\right.
$$

for some fixed constants $c_{i}$ satisfying $1<c_{0}<c_{i}(i=1,2, \ldots, 6)$ and some time $T_{2}>0$. Here

$$
c_{0}=2+\left\|\left(\rho_{0}, u_{0}\right)\right\|_{H^{3}}+\left\|\left(h_{0}, k_{0}, \varepsilon_{0}\right)\right\|_{H^{2}} .
$$

For simplicity, we set another small time $T$ as $T=\min \left\{c_{0}^{-6 \gamma-16} c_{1}^{-10} c_{2}^{-8} c_{3}^{-8} c_{4}^{-2} c_{5}^{-2} c_{6}^{-4}, T_{1}, T_{2}\right\}$ and all of the $T$ in Section 2 are defined as this.

Remark 2.1 Here it should be emphasized that throughout this paper, $C$ denotes a generic positive constant which is only dependent on $m, \gamma$, and $|\Omega|$, but independent of $c_{i}(i=$ $0,1,2, \ldots, 6)$.

Remark 2.2 From the physical viewpoint, we assume that the turbulent kinetic energy $k$ has a positive lower bound away from zero, namely, $0<m<k$ with $m$ a constant. We do not know whether $0<m<k$ holds afterwards if the initial turbulent kinetic energy $k_{0}>m$.

In this section we aim to prove the following local existence theorem of the linearized system (2.2)-(2.6). 
Theorem 2.1 There exists a unique strong solution $(\rho, u, h, k, \varepsilon)$ to the linearized problem (2.2)-(2.8) and (2.9) in [0,T] satisfying the estimates (2.99) and (2.100) as well as the regularity

$$
\begin{aligned}
& \rho \in C\left(0, T ; H^{3}\right), \quad \rho_{t} \in C\left(0, T ; H^{1}\right), \quad u \in C\left(0, T ; H^{3}\right) \cap L^{2}\left(0, T ; H^{4}\right), \\
& u_{t} \in L^{2}\left(0, T ; H^{1}\right), \quad k \in C\left(0, T ; H^{2}\right) \cap L^{2}\left(0, T ; H^{3}\right), \quad k_{t} \in L^{2}\left(0, T ; H^{1}\right), \\
& \varepsilon \in C\left(0, T ; H^{2}\right), \quad \varepsilon_{t} \in L^{2}\left(0, T ; H^{1}\right), \quad h \in C\left(0, T ; H^{2}\right), \quad h_{t} \in L^{2}\left(0, T ; H^{1}\right), \\
& \left(\sqrt{\rho} u_{t}, \sqrt{\rho} k_{t}, \sqrt{\rho} \varepsilon_{t}, \sqrt{\rho} h_{t}\right) \in L^{\infty}\left(0, T ; L^{2}\right) .
\end{aligned}
$$

In the following part, we decompose the proof of Theorem 2.1 into some lemmas.

Lemma 2.1 There exists a unique strong solution $\rho$ to the linear transport problem (2.2) and (2.9) such that

$$
\rho \geq \frac{m}{e}, \quad\|\rho\|_{H^{3}(\Omega)} \leq C c_{0}, \quad\left\|\rho_{t}\right\|_{H^{1}(\Omega)} \leq C c_{0} c_{2}
$$

for $0 \leq t \leq T$.

Proof First, applying the particle trajectory method to the equation (2.3), we easily deduce

$$
\rho \geq \rho_{0} \exp \left(-\int_{0}^{T}\|\nabla v\|_{L^{\infty}} \mathrm{d} t\right) \geq \rho_{0} \exp \left(-c_{3} T\right) \geq \frac{\rho_{0}}{e} \geq \frac{m}{e}
$$

and thus

$$
\frac{1}{\rho} \leq \frac{e}{m} \leq C
$$

Second, by simple calculation, we have

$$
\frac{\mathrm{d}}{\mathrm{d} t}\|\rho\|_{H^{3}} \leq C\|v\|_{H^{3}}\|\rho\|_{H^{3}}+C\left\|\nabla^{4} v\right\|_{L^{2}}
$$

applying the Gronwall and Hölder's inequalities, one gets

$$
\|\rho\|_{H^{3}} \leq\left[\exp \left(C \int_{0}^{t}\|v\|_{H^{3}} \mathrm{~d} t\right)\right]\left(\left\|\rho_{0}\right\|_{H^{3}}+C \int_{0}^{t}\|v\|_{H^{4}} \mathrm{~d} t\right) \leq C c_{0}
$$

for $0 \leq t \leq T$.

Next, from the equation (2.2), one obtains

$$
\left\|\rho_{t}\right\|_{H^{1}}=\|\nabla \cdot(\rho v)\|_{H^{1}} \leq C\|\rho\|_{H^{3}}\|v\|_{H^{2}} \leq C c_{0} c_{2}
$$

for $0 \leq t \leq T$.

Thus, we complete the proof of Lemma 2.1.

Next, we estimate the velocity field $u$. 
Lemma 2.2 There exists a unique strong solution $u$ to the initial boundary value problem (2.3) and (2.9) such that

$$
\begin{aligned}
& \left\|\sqrt{\rho} u_{t}\right\|_{L^{2}}^{2}+\|u\|_{H^{1}}^{2}+\int_{0}^{t}\left\|\nabla u_{t}\right\|_{L^{2}}^{2} \mathrm{~d} s \leq C c_{0}^{5+2 \gamma}, \quad\|u\|_{H^{2}} \leq C c_{0}^{\frac{5}{2}+3 \gamma} c_{1}^{2}, \\
& \|u\|_{H^{3}} \leq C c_{0}^{\frac{13}{2}+3 \gamma} c_{1}^{4} c_{2} c_{5}, \quad \int_{0}^{t}\|u\|_{H^{4}}^{2} \mathrm{~d} s \leq C c_{0}^{9+6 \gamma} c_{1}^{5} c_{2}^{2}
\end{aligned}
$$

for $0 \leq t \leq T$.

Proof We only need to prove the estimates. Differentiating the equation (2.3) with respect to $t$, then multiplying both sides of the result by $u_{t}$ and integrating over $\Omega$, we derive that

$$
\begin{aligned}
\frac{1}{2} & \frac{\mathrm{d}}{\mathrm{d} t} \int \rho u_{t}^{2} \mathrm{~d} x+\left\|\nabla u_{t}\right\|_{L^{2}}^{2}+\left\|\operatorname{div} u_{t}\right\|_{L^{2}}^{2} \\
= & -\int \rho_{t} \nu \cdot \nabla u \cdot u_{t}-\int \rho v_{t} \cdot \nabla u \cdot u_{t}-2 \int \rho v \cdot \nabla u_{t} \cdot u_{t} \\
& \quad-\int \nabla p_{t} \cdot u_{t}-\frac{2}{3} \int[\nabla(\rho \pi)]_{t} \cdot u_{t} \\
= & I_{1}+I_{2}+I_{3}+I_{4}+I_{5},
\end{aligned}
$$

where we have used the equation (2.2) and integration by parts. We will estimate $I_{i}(i=$ $1,2, \ldots, 5)$ item by item.

First, because $\rho$ has a lower bound away from zero, we easily deduce $\left\|u_{t}\right\|_{L^{2}} \leq$ $C\left\|\sqrt{\rho} u_{t}\right\|_{L^{2}}$. Therefore, using the Hölder, Sobolev, and Young inequalities and (2.10), we have

$$
\begin{aligned}
I_{1} & \leq C\|v\|_{L^{\infty}}\left\|\rho_{t}\right\|_{L^{3}}\|\nabla u\|_{L^{2}}\left\|u_{t}\right\|_{L^{6}} \leq C\|v\|_{L^{\infty}}\left\|\rho_{t}\right\|_{L^{3}}\|\nabla u\|_{L^{2}}\left(\left\|\sqrt{\rho} u_{t}\right\|_{L^{2}}+\left\|\nabla u_{t}\right\|_{L^{2}}\right) \\
& \leq C c_{0}^{2} c_{2}^{4}\|\nabla u\|_{L^{2}}^{2}+C\left\|\sqrt{\rho} u_{t}\right\|_{L^{2}}^{2}+\frac{1}{8}\left\|\nabla u_{t}\right\|_{L^{2}}^{2} \\
I_{3} & \leq C\|\rho\|_{L^{\infty}}^{\frac{1}{2}}\|v\|_{L^{\infty}}\left\|\nabla u_{t}\right\|_{L^{2}}\left\|\sqrt{\rho} u_{t}\right\|_{L^{2}} \leq C c_{0} c_{2}^{2}\left\|\sqrt{\rho} u_{t}\right\|_{L^{2}}^{2}+\frac{1}{8}\left\|\nabla u_{t}\right\|_{L^{2}}^{2} \\
I_{2} & \leq C\|\rho\|_{L^{\infty}}^{\frac{1}{2}}\left\|v_{t}\right\|_{L^{6}}\|\nabla u\|_{L^{3}}\left\|\sqrt{\rho} u_{t}\right\|_{L^{2}} \leq C \eta^{-1} c_{0}\|\nabla u\|_{L^{3}}^{2}+\eta\left\|v_{t}\right\|_{H^{1}}^{2}\left\|\sqrt{\rho} u_{t}\right\|_{L^{2}}^{2}
\end{aligned}
$$

where $\eta>0$ is a small number to be determined later.

Next, to evaluate $\|\nabla u\|_{L^{3}}^{2}$ in (2.17), we can first the Sobolev interpolation inequality to get

$$
\|\nabla u\|_{L^{3}}^{2} \leq C\|\nabla u\|_{L^{2}}\|\nabla u\|_{L^{6}} \leq C\|\nabla u\|_{L^{2}}\|\nabla u\|_{H^{1}} .
$$

Then applying the standard elliptic regularity result to the equation (2.3) and using (2.18), we have

$$
\begin{aligned}
\|\nabla u\|_{H^{1}} \leq & C c_{0}^{\gamma}\left(\left\|\sqrt{\rho} u_{t}\right\|_{L^{2}}+\|v\|_{L^{6}}\|\nabla u\|_{L^{2}}^{\frac{1}{2}}\|\nabla u\|_{H^{1}}^{\frac{1}{2}}+\|\nabla \rho\|_{L^{2}}\right. \\
& \left.+\|\nabla \rho\|_{L^{4}}\|\pi\|_{L^{4}}+\|\nabla \pi\|_{L^{2}}\right)
\end{aligned}
$$


thus the Young inequality and (2.10) yield

$$
\|\nabla u\|_{H^{1}} \leq C c_{0}^{2 \gamma}\left(\left\|\sqrt{\rho} u_{t}\right\|_{L^{2}}+c_{1}^{2}\|\nabla u\|_{L^{2}}+c_{0} c_{1}\right) .
$$

Combining (2.17), (2.18), and (2.19), and using the Young inequality, we get

$$
I_{2} \leq C \eta^{-1} c_{0}^{2 \gamma+1}\left(\left\|\sqrt{\rho} u_{t}\right\|_{L^{2}}^{2}+c_{1}^{2}\|\nabla u\|_{L^{2}}^{2}+c_{0}^{2} c_{1}^{2}\right)+\eta\left\|v_{t}\right\|_{H^{1}}^{2}\left\|\sqrt{\rho} u_{t}\right\|_{L^{2}}^{2} .
$$

By integration by parts, we have

$$
\begin{aligned}
I_{4} & =\int p_{t} \operatorname{div} u_{t} \leq C c_{0}^{\gamma-1}\left\|\rho_{t}\right\|_{L^{2}}\left\|\nabla u_{t}\right\|_{L^{2}} \leq C c_{0}^{2 \gamma} c_{2}^{2}+\frac{1}{8}\left\|\nabla u_{t}\right\|_{L^{2}}^{2} \\
I_{5} & =\frac{2}{3} \int \rho_{t} \pi \nabla \cdot u_{t}-\frac{2}{3} \int \pi_{t} \nabla \rho \cdot u_{t}-\frac{2}{3} \int \rho \nabla \pi_{t} \cdot u_{t} \\
& \leq C\left\|\rho_{t}\right\|_{L^{3}}\|\pi\|_{L^{6}}\left\|\nabla u_{t}\right\|_{L^{2}}+C c_{0}^{\frac{1}{2}}\|\nabla \rho\|_{L^{3}}\left\|\pi_{t}\right\|_{L^{6}}\left\|\sqrt{\rho} u_{t}\right\|_{L^{2}}+C c_{0}^{\frac{1}{2}}\left\|\nabla \pi_{t}\right\|_{L^{2}}\left\|\sqrt{\rho} u_{t}\right\|_{L^{2}} \\
& \leq C c_{0}^{2} c_{1}^{2} c_{2}^{2}+C \eta^{-1} c_{0}^{3}+C \eta\left\|\pi_{t}\right\|_{H^{1}}^{2}\left\|\sqrt{\rho} u_{t}\right\|_{L^{2}}^{2}+\frac{1}{8}\left\|\nabla u_{t}\right\|_{L^{2}}^{2}
\end{aligned}
$$

On the other hand, we easily have

$$
\frac{\mathrm{d}}{\mathrm{d} t} \int|\nabla u|^{2}=2 \int \nabla u \cdot \nabla u_{t} \leq \frac{1}{8}\left\|\nabla u_{t}\right\|_{L^{2}}^{2}+C\|\nabla u\|_{L^{2}}^{2}
$$

and

$$
\frac{\mathrm{d}}{\mathrm{d} t} \int|u|^{2} \leq C c_{0}^{\frac{1}{2}}\left\|\sqrt{\rho} u_{t}\right\|_{L^{2}}\|u\|_{L^{2}} \leq C c_{0}\left\|\sqrt{\rho} u_{t}\right\|_{L^{2}}^{2}+C\|u\|_{L^{2}}^{2} .
$$

Combining (2.14)-(2.16) and (2.20)-(2.24), we get

$$
\begin{aligned}
\frac{\mathrm{d}}{\mathrm{d} t}( & \left.\left\|\sqrt{\rho} u_{t}\right\|_{L^{2}}^{2}+\|u\|_{H^{1}}^{2}\right)+\left\|\nabla u_{t}\right\|_{L^{2}}^{2} \\
\leq & C\left(c_{0}^{2} c_{2}^{4}+\eta^{-1} c_{0}^{2 \gamma+1} c_{1}^{2}+\eta\left\|\pi_{t}\right\|_{H^{1}}^{2}+\eta\left\|v_{t}\right\|_{H^{1}}^{2}\right)\left(\left\|\sqrt{\rho} u_{t}\right\|_{L^{2}}^{2}+\|u\|_{H^{1}}^{2}\right) \\
& +C\left(c_{0}^{2 \gamma} c_{1}^{2} c_{2}^{2}+\eta^{-1} c_{0}^{2 \gamma+3} c_{1}^{2}\right),
\end{aligned}
$$

setting $\eta=\frac{1}{c_{1}}$ and using the Gronwall inequality, we derive

$$
\left\|\sqrt{\rho} u_{t}\right\|_{L^{2}}^{2}+\|u\|_{H^{1}}^{2}+\int_{0}^{t}\left\|\nabla u_{t}\right\|_{L^{2}}^{2} \mathrm{~d} s \leq C c_{0}^{5+2 \gamma}
$$

for $0 \leq t \leq T$, where we have used the fact that $\lim _{t \rightarrow 0}\left(\left\|\sqrt{\rho} u_{t}\right\|_{L^{2}}^{2}+\|u\|_{H^{1}}^{2}\right) \leq C c_{0}^{5+2 \gamma}$.

Next, by (2.19) and (2.26), we deduce

$$
\|\nabla u\|_{H^{1}} \leq C c_{0}^{\frac{5}{2}+3 \gamma} c_{1}^{2}
$$

which implies (2.12) by (2.26). 
Next, we will estimate $\int_{0}^{t}\|u\|_{H^{4}}^{2} \mathrm{~d} t$. By the standard elliptic regularity result of the equation (2.3), we have

$$
\left\|\nabla^{4} u\right\|_{L^{2}} \leq\left\|\rho u_{t}\right\|_{H^{2}}+\|\rho v \cdot \nabla u\|_{H^{2}}+\|\nabla p\|_{H^{2}}+\left\|\frac{2}{3} \nabla(\rho \pi)\right\|_{H^{2}} .
$$

By simple calculation, the first term of the right-hand side of (2.28) can be controlled as

$$
\left\|\rho u_{t}\right\|_{H^{2}} \leq C\left(\left\|\rho u_{t}\right\|_{L^{2}}+\|\rho\|_{H^{2}}\left\|u_{t}\right\|_{H^{2}}\right) \leq C c_{0}\left\|u_{t}\right\|_{H^{2}} .
$$

In order to estimate $\left\|\nabla^{2} u_{t}\right\|_{L^{2}}$, differentiating the equation (2.3) with respect to $t$ yields

$$
\begin{aligned}
\Delta u_{t}+\nabla \operatorname{div} u_{t}= & \rho_{t} u_{t}+\rho u_{t t}+\rho_{t} v \cdot \nabla u+\rho v_{t} \cdot \nabla u+\rho v \cdot \nabla u_{t}+\nabla p_{t} \\
& +\frac{2}{3}\left(\nabla \rho_{t} \pi+\rho_{t} \nabla \pi+\nabla \rho \pi_{t}+\rho \nabla \pi_{t}\right),
\end{aligned}
$$

applying the standard elliptic regularity result to (2.30) and using (2.26), one obtains

$$
\begin{aligned}
\left\|\nabla^{2} u_{t}\right\|_{L^{2}} \leq & C\left(\left\|\rho_{t}\right\|_{L^{4}}\left\|u_{t}\right\|_{L^{4}}+\left\|\rho u_{t t}\right\|_{L^{2}}+\left\|\rho_{t}\right\|_{L^{4}}\|v\|_{L^{\infty}}\|\nabla u\|_{L^{4}}\right. \\
& +\|\rho\|_{L^{\infty}}\left\|v_{t}\right\|_{L^{4}}\|\nabla u\|_{L^{4}}+\|v\|_{L^{\infty}}\left\|u_{t}\right\|_{H^{1}}+\|\rho\|_{H^{2}}^{\gamma}\left\|\rho_{t}\right\|_{H^{1}}+\|\pi\|_{L^{\infty}}\left\|\rho_{t}\right\|_{H^{1}} \\
& \left.+\left\|\rho_{t}\right\|_{L^{4}}\|\nabla \pi\|_{L^{4}}+\|\nabla \rho\|_{L^{4}}\left\|\pi_{t}\right\|_{L^{4}}+\|\rho\|_{L^{\infty}}\left\|\nabla \pi_{t}\right\|_{L^{2}}\right) \\
\leq & C\left(\left\|\rho u_{t t}\right\|_{L^{2}}+c_{0}^{\frac{7}{2}+3 \gamma} c_{1}^{2} c_{2}^{2} c_{5}+c_{0}^{\frac{7}{2}+3 \gamma} c_{1}^{2}\left\|v_{t}\right\|_{H^{1}}\right. \\
& \left.+c_{0} c_{2}\left\|u_{t}\right\|_{H^{1}}+c_{0}\left\|\pi_{t}\right\|_{H^{1}}\right)
\end{aligned}
$$

therefore, the key point is to estimate $\left\|\rho u_{t t}\right\|_{L^{2}}$. Because we have the fact $\left\|\rho u_{t t}\right\|_{L^{2}} \leq$ $C\left\|\sqrt{\rho} u_{t t}\right\|_{L^{2}}$, we could first estimate $\left\|\sqrt{\rho} u_{t t}\right\|_{L^{2}}$ as follows.

Multiplying both sides of (2.30) by $u_{t t}$ and integrating the result over $\Omega$ yield

$$
\begin{aligned}
\int & \rho u_{t t}^{2} \mathrm{~d} x+\frac{1}{2} \frac{\mathrm{d}}{\mathrm{d} t}\left\|\nabla u_{t}\right\|_{L^{2}}^{2}+\frac{1}{2} \frac{\mathrm{d}}{\mathrm{d} t}\left\|\operatorname{div} u_{t}\right\|_{L^{2}}^{2} \\
= & -\int \rho_{t} u_{t} \cdot u_{t t}-\int \rho_{t} v \cdot \nabla u \cdot u_{t t}-\int \rho v_{t} \cdot \nabla u \cdot u_{t t}-\int \rho v \cdot \nabla u_{t} \cdot u_{t t}-\int \nabla p_{t} \cdot u_{t t} \\
& \quad-\frac{2}{3} \int\left(\pi \nabla \rho_{t}+\rho_{t} \nabla \pi+\pi_{t} \nabla \rho+\rho \nabla \pi_{t}\right) \cdot u_{t t} \\
= & J_{1}+J_{2}+J_{3}+J_{4}+J_{5}+J_{6} .
\end{aligned}
$$

Using the Hölder, Sobolev, and Young inequalities and (2.10) and (2.26), we get

$$
\begin{aligned}
J_{1} & \leq C c_{0}^{\frac{1}{2}}\left\|\rho_{t}\right\|_{L^{3}}\left\|u_{t}\right\|_{L^{6}}\left\|\sqrt{\rho} u_{t t}\right\|_{L^{2}} \leq C c_{0}^{\frac{1}{2}}\left\|\rho_{t}\right\|_{L^{3}}\left(\left\|\sqrt{\rho} u_{t}\right\|_{L^{2}}+\left\|\nabla u_{t}\right\|_{L^{2}}\right)\left\|\sqrt{\rho} u_{t t}\right\|_{L^{2}} \\
& \leq C c_{0}^{3} c_{2}^{2}\left\|\nabla u_{t}\right\|_{L^{2}}^{2}+C c_{0}^{8+2 \gamma} c_{2}^{2}+\frac{1}{18}\left\|\sqrt{\rho} u_{t t}\right\|_{L^{2}}^{2} \\
J_{2} & \leq C c_{0}^{\frac{1}{2}}\left\|\sqrt{\rho} u_{t t}\right\|_{L^{2}}\left\|\rho_{t}\right\|_{L^{3}}\|v\|_{L^{\infty}}\|\nabla u\|_{L^{6}} \leq C c_{0}^{8+6 \gamma} c_{1}^{4} c_{2}^{4}+\frac{1}{18}\left\|\sqrt{\rho} u_{t t}\right\|_{L^{2}}^{2} \\
J_{3} & \leq C c_{0}^{\frac{1}{2}}\left\|\sqrt{\rho} u_{t t}\right\|_{L^{2}}\left\|v_{t}\right\|_{L^{3}}\|\nabla u\|_{L^{6}} \leq C c_{0}^{6+6 \gamma} c_{1}^{4}\left\|v_{t}\right\|_{H^{1}}^{2}+\frac{1}{18}\left\|\sqrt{\rho} u_{t t}\right\|_{L^{2}}^{2}
\end{aligned}
$$




$$
\begin{aligned}
J_{4} \leq & C c_{0}^{\frac{1}{2}}\|v\|_{L^{\infty}}\left\|\sqrt{\rho} u_{t t}\right\|_{L^{2}}\left\|\nabla u_{t}\right\|_{L^{2}} \leq C c_{0} c_{2}^{2}\left\|\nabla u_{t}\right\|_{L^{2}}^{2}+\frac{1}{18}\left\|\sqrt{\rho} u_{t t}\right\|_{L^{2}}^{2}, \\
J_{5} \leq & C c_{0}^{\frac{1}{2}}\left\|\sqrt{\rho} u_{t t}\right\|_{L^{2}}\left\|\nabla p_{t}\right\|_{L^{2}} \leq C c_{0}^{2 \gamma+1} c_{2}^{2}+\frac{1}{18}\left\|\sqrt{\rho} u_{t t}\right\|_{L^{2}}^{2}, \\
J_{6} \leq & C c_{0}^{\frac{1}{2}}\|\pi\|_{L^{\infty}}\left\|\sqrt{\rho} u_{t t}\right\|_{L^{2}}\left\|\nabla \rho_{t}\right\|_{L^{2}}+C c_{0}^{\frac{1}{2}}\left\|\sqrt{\rho} u_{t t}\right\|_{L^{2}}\|\nabla \pi\|_{L^{4}}\left\|\rho_{t}\right\|_{L^{4}} \\
& +C c_{0}^{\frac{1}{2}}\left\|\sqrt{\rho} u_{t t}\right\|_{L^{2}}\|\nabla \rho\|_{L^{\infty}}\left\|\pi_{t}\right\|_{L^{2}}+C c_{0}^{\frac{1}{2}}\left\|\sqrt{\rho} u_{t t}\right\|_{L^{2}}\left\|\nabla \pi_{t}\right\|_{L^{2}} \\
\leq & C c_{0}^{3} c_{2}^{2} c_{5}^{2}+C c_{0}^{3}\left\|\pi_{t}\right\|_{H^{1}}^{2}+\frac{2}{9}\left\|\sqrt{\rho} u_{t t}\right\|_{L^{2}}^{2},
\end{aligned}
$$

inserting (2.33)-(2.38) to (2.32), then integrating the result over $(0, t)$, we derive

$$
\int_{0}^{t} \int_{\Omega} \rho u_{t t}^{2} \mathrm{~d} x \mathrm{~d} t+\left\|\nabla u_{t}\right\|_{L^{2}}^{2} \leq C c_{0}^{6+6 \gamma} c_{1}^{5} c_{2}^{2}
$$

where we have used the equation (2.3) to get $\lim _{t \rightarrow 0}\left\|\nabla u_{t}(t)\right\|_{L^{2}}^{2} \leq C c_{0}^{2 \gamma+4}$.

So, combining (2.29), (2.31), and (2.39), we obtain

$$
\int_{0}^{t}\left\|\rho u_{t}\right\|_{H^{2}}^{2} \leq C c_{0}^{9+6 \gamma} c_{1}^{5} c_{2}^{2}
$$

In the following, we shall estimate the rest terms of the inequality (2.28).

For the second term of the inequality (2.28), direct calculation yields

$$
\|\rho v \cdot \nabla u\|_{H^{2}} \leq C\|\rho\|_{H^{2}}\|v\|_{H^{2}}\|u\|_{H^{3}} \leq C c_{0} c_{2}\|u\|_{H^{3}}
$$

therefore, we have to evaluate $\|u\|_{H^{3}}$. In fact, applying the standard elliptic regularity result to the equation (2.3), we obtain

$$
\left\|\nabla^{3} u\right\|_{L^{2}} \leq C\left(\left\|\rho u_{t}\right\|_{H^{1}}+\|\rho v \cdot \nabla u\|_{H^{1}}+\|\nabla p\|_{H^{1}}+\|\nabla(\rho \pi)\|_{H^{1}}\right)
$$

we could estimate the right-hand side of (2.42) item by item.

First, from (2.26), we have $\left\|u_{t}\right\|_{L^{2}} \leq C c_{0}^{\frac{5}{2}+\gamma}$, thus

$$
\begin{aligned}
\left\|\rho u_{t}\right\|_{H^{1}} & \leq C c_{0}\left\|u_{t}\right\|_{L^{2}}+\|\nabla \rho\|_{L^{\infty}}\left\|u_{t}\right\|_{L^{2}}+C c_{0}\left\|\nabla u_{t}\right\|_{L^{2}} \\
& \leq C c_{0}^{\frac{7}{2}+\gamma}+C c_{0}\left\|\nabla u_{t}\right\|_{L^{2}} .
\end{aligned}
$$

Second, using the Sobolev interpolation inequality and the Young inequality, we get

$$
\begin{aligned}
\|\rho v \cdot \nabla u\|_{H^{1}} \\
\leq C\left(\|\rho v \cdot \nabla u\|_{L^{2}}+\|\nabla(\rho v \cdot \nabla u)\|_{L^{2}}\right) \\
\leq C\left(c_{0}\|v\|_{L^{\infty}}\|\nabla u\|_{L^{2}}+\|\nabla \rho\|_{L^{\infty}}\|v\|_{L^{\infty}}\|\nabla u\|_{L^{2}}+c_{0}\|\nabla v\|_{L^{2}}\|\nabla u\|_{L^{2}}^{\frac{1}{4}}\left\|\nabla^{3} u\right\|_{L^{2}}^{\frac{3}{4}}\right. \\
\left.\quad+c_{0}\|v\|_{L^{\infty}}\left\|\nabla^{2} u\right\|_{L^{2}}\right) \\
\leq C c_{0}^{\frac{13}{2}+3 \gamma} c_{1}^{4} c_{2}+\frac{3}{4}\|u\|_{H^{3}} .
\end{aligned}
$$


Third, due to (2.11), we easily derive

$$
\|\nabla p\|_{H^{1}} \leq C c_{0}^{2} .
$$

Last, by simple calculation, one gets

$$
\|\nabla(\rho \pi)\|_{H^{1}} \leq C\|\rho\|_{H^{3}}\|\pi\|_{H^{2}} \leq C c_{0} c_{5} .
$$

Combining (2.39) and (2.42)-(2.46), we deduce

$$
\|u\|_{H^{3}} \leq C c_{0}^{\frac{13}{2}+3 \gamma} c_{1}^{4} c_{2} c_{5} .
$$

Next, by simple calculation, the third and fourth terms on the right-hand side of (2.28) can be estimated as

$$
\|\nabla p\|_{H^{2}} \leq C c_{0}^{3}, \quad\|\nabla(\rho \pi)\|_{H^{2}} \leq C c_{0}\|\pi\|_{H^{3}}
$$

Combining (2.26), (2.28), (2.40), (2.41), and (2.47)-(2.48), one deduces

$$
\int_{0}^{t}\|u\|_{H^{4}}^{2} \mathrm{~d} t \leq C c_{0}^{9+6 \gamma} c_{1}^{5} c_{2}^{2}
$$

for $0 \leq t \leq T$.

Thus, we complete the proof of Lemma 2.2 .

In the following part, we estimate the turbulent kinetic energy $k$.

Lemma 2.3 There exists a unique strong solution $k$ to the initial boundary value problem (2.5) and (2.9) such that

$$
\begin{aligned}
& \left\|\sqrt{\rho} k_{t}\right\|_{L^{2}}^{2}+\|k\|_{H^{1}}^{2}+\int_{0}^{t}\left\|\nabla k_{t}\right\|_{L^{2}}^{2} \mathrm{~d} s \leq C c_{0}^{5}, \\
& \|k\|_{H^{2}} \leq C c_{0}^{\frac{7}{2}} c_{1} c_{2}^{2}, \quad \int_{0}^{t}\|k\|_{H^{3}}^{2} \mathrm{~d} s \leq C c_{0}^{7},
\end{aligned}
$$

for $0 \leq t \leq T$.

Proof We only need to prove the estimates. Differentiating the equation (2.5) with respect to $t$, then multiplying both sides of the resulting equation by $k_{t}$ and integrating over $\Omega$, we get

$$
\begin{aligned}
\frac{1 \mathrm{~d}}{\mathrm{~d} t}\left\|\sqrt{\rho} k_{t}\right\|_{L^{2}}^{2}+\left\|\nabla k_{t}\right\|_{L^{2}}^{2}= & -\int \rho_{t} \nu \cdot \nabla k \cdot k_{t}-\int \rho v_{t} \cdot \nabla k \cdot k_{t}-2 \int \rho \nu \cdot \nabla k_{t} \cdot k_{t} \\
& +\int G_{t}^{\prime} \cdot k_{t}-\int \rho_{t} \theta \cdot k_{t}-\int \rho \theta_{t} \cdot k_{t} \\
= & \sum_{i=1}^{6} K_{i},
\end{aligned}
$$

we could evaluate $K_{i}(i=1, \ldots, 6)$ as follows. 
First, using a similar method to deriving (2.15), (2.20), (2.16), respectively, one has

$$
\begin{aligned}
& K_{1} \leq C c_{0}^{2} c_{2}^{4}\|\nabla k\|_{L^{2}}^{2}+C\left\|\sqrt{\rho} k_{t}\right\|_{L^{2}}^{2}+\frac{1}{10}\left\|\nabla k_{t}\right\|_{L^{2}}^{2}, \\
& K_{2} \leq C \eta^{-1} c_{0}^{2 \gamma+1}\left(\left\|\sqrt{\rho} k_{t}\right\|_{L^{2}}^{2}+c_{1}^{2}\|\nabla k\|_{L^{2}}^{2}+c_{0}^{2} c_{1}^{2} c_{2}^{4}\right)+\eta\left\|v_{t}\right\|_{H^{1}}^{2}\left\|\sqrt{\rho} k_{t}\right\|_{L^{2}}^{2}, \\
& K_{3} \leq C c_{0} c_{2}^{2}\left\|\sqrt{\rho} k_{t}\right\|_{L^{2}}^{2}+\frac{1}{10}\left\|\nabla k_{t}\right\|_{L^{2}}^{2} .
\end{aligned}
$$

Next, differentiating $G^{\prime}$ with respect to $t$ and inserting the result thus obtained into $K_{4}$ yield

$$
\begin{aligned}
K_{4} \leq & C \int\left|\nabla v_{t}\right||\nabla v|\left|k_{t}\right|+C \int|\rho||\pi|\left|\nabla v_{t}\right|\left|k_{t}\right|+C \int\left|\rho_{t}\right||\pi|\left|\nabla v \| k_{t}\right| \\
& +C \int\left|\rho\left\|\pi_{t}|| \nabla v\right\| k_{t}\right| \\
\leq & C c_{0}^{\frac{1}{2}}\left\|\sqrt{\rho} k_{t}\right\|_{L^{2}}\left\|\nabla v_{t}\right\|_{L^{2}}\|\nabla v\|_{L^{\infty}}+C c_{0}^{\frac{1}{2}}\|\pi\|_{L^{\infty}}\left\|\nabla v_{t}\right\|_{L^{2}}\left\|\sqrt{\rho} k_{t}\right\|_{L^{2}} \\
& +C\|\pi\|_{L^{\infty}}\left\|\rho_{t}\right\|_{L^{3}}\|\nabla v\|_{L^{2}}\left\|k_{t}\right\|_{L^{6}}+C c_{0}^{\frac{1}{2}}\left\|\sqrt{\rho} k_{t}\right\|_{L^{2}}\left\|\pi_{t}\right\|_{L^{6}}\|\nabla v\|_{L^{3}} \\
\leq & C \eta^{-1} c_{0} c_{3}^{2} c_{5}^{2}+C c_{0}^{2} c_{1}^{2} c_{2}^{2} c_{5}^{2}+C\left\|^{\rho} k_{t}\right\|_{L^{2}}^{2}+C \eta\left(\left\|v_{t}\right\|_{H^{1}}^{2}+\left\|\pi_{t}\right\|_{H^{1}}^{2}\right)\left\|\sqrt{\rho} k_{t}\right\|_{L^{2}}^{2} \\
& +\frac{1}{10}\left\|\nabla k_{t}\right\|_{L^{2}}^{2} .
\end{aligned}
$$

Last, direct calculation leads to

$$
\begin{aligned}
& K_{5} \leq\left\|\rho_{t}\right\|_{L^{3}}\|\theta\|_{L^{2}}\left\|k_{t}\right\|_{L^{6}} \leq C c_{0}^{2} c_{1}^{2} c_{2}^{2}+C\left\|\sqrt{\rho} k_{t}\right\|_{L^{2}}^{2}+\frac{1}{10}\left\|\nabla k_{t}\right\|_{L^{2}}^{2}, \\
& K_{6} \leq C c_{0}^{\frac{1}{2}}\left\|\sqrt{\rho} k_{t}\right\|_{L^{2}}\left\|\theta_{t}\right\|_{L^{2}} \leq C \eta^{-1} c_{0}+\eta\left\|\theta_{t}\right\|_{L^{2}}^{2}\left\|\sqrt{\rho} k_{t}\right\|_{L^{2}}^{2} .
\end{aligned}
$$

On the other hand, we easily get

$$
\begin{aligned}
& \frac{\mathrm{d}}{\mathrm{d} t}\|\nabla k\|_{L^{2}}^{2} \leq \frac{1}{10}\left\|\nabla k_{t}\right\|_{L^{2}}^{2}+C\|\nabla k\|_{L^{2}}^{2}, \\
& \frac{\mathrm{d}}{\mathrm{d} t}\|k\|_{L^{2}}^{2} \leq C c_{0}\left\|\sqrt{\rho} k_{t}\right\|_{L^{2}}^{2}+C\|k\|_{L^{2}}^{2} .
\end{aligned}
$$

Combining (2.52)-(2.60), we obtain

$$
\begin{aligned}
& \frac{\mathrm{d}}{\mathrm{d} t}\left(\left\|\sqrt{\rho} k_{t}\right\|_{L^{2}}^{2}+\|k\|_{H^{1}}^{2}\right)+\left\|\nabla k_{t}\right\|_{L^{2}}^{2} \\
& \leq C\left(c_{0}^{2} c_{2}^{4}+\eta^{-1} c_{0}^{2 \gamma+1} c_{1}^{2}+\eta\left\|v_{t}\right\|_{H^{1}}^{2}+\eta\left\|\pi_{t}\right\|_{H^{1}}^{2}+\eta\left\|\theta_{t}\right\|_{L^{2}}^{2}\right)\left(\left\|\sqrt{\rho} k_{t}\right\|_{L^{2}}^{2}+\|k\|_{H^{1}}^{2}\right) \\
& \quad+C\left(\eta^{-1} c_{0}^{2} c_{1}^{2} c_{2}^{4} c_{3}^{2} c_{5}^{2}+c_{0}^{2} c_{1}^{2} c_{2}^{2} c_{5}^{2}\right),
\end{aligned}
$$

setting $\eta=c_{1}^{-1}$ and using the Gronwall inequality, we deduce

$$
\left\|\sqrt{\rho} k_{t}\right\|_{L^{2}}^{2}+\|k\|_{H^{1}}^{2}+\int_{0}^{t}\left\|\nabla k_{t}\right\|_{L^{2}}^{2} \mathrm{~d} s \leq C c_{0}^{5}
$$

for $0 \leq t \leq T$, where we have used the fact that $\lim _{t \rightarrow 0}\left(\left\|\sqrt{\rho} k_{t}\right\|_{L^{2}}^{2}+\|k\|_{H^{1}}^{2}\right) \leq C c_{0}^{5}$. 
Then, by the standard elliptic regularity result of the equation (2.5) and using (2.62), we have

$$
\begin{aligned}
\|\nabla k\|_{H^{1}} \leq & C c_{0}^{\frac{1}{2}}\left\|\sqrt{\rho} k_{t}\right\|_{L^{2}}+C c_{0}\|v\|_{L^{\infty}}\|\nabla k\|_{L^{2}}+C\|\nabla v\|_{L^{4}}^{2} \\
& +C c_{0}\|\pi\|_{L^{4}}\|\nabla v\|_{L^{4}}+C c_{0}\|\theta\|_{L^{2}} \\
\leq & C c_{0}^{\frac{7}{2}} c_{1} c_{2}^{2}
\end{aligned}
$$

and

$$
\left\|\nabla^{2} k\right\|_{H^{1}} \leq C\left(\left\|\rho k_{t}\right\|_{H^{1}}+\|\rho v \cdot \nabla k\|_{H^{1}}+\left\|G^{\prime}\right\|_{H^{1}}+\|\rho \theta\|_{H^{1}}\right) .
$$

To evaluate $\int_{0}^{t}\|k\|_{H^{3}}^{2} \mathrm{~d} t$, we will estimate the right-hand side of (2.64) item by item. In fact, we derive by using (2.62) and (2.63) that

$$
\begin{aligned}
&\left\|\rho k_{t}\right\|_{H^{1}} \leq C\left(\left\|\rho k_{t}\right\|_{L^{2}}+\left\|\nabla\left(\rho k_{t}\right)\right\|_{L^{2}}\right) \\
& \leq C c_{0}^{\frac{7}{2}}+C c_{0}\left\|\nabla k_{t}\right\|_{L^{2}}, \\
&\|\rho v \cdot \nabla k\|_{H^{1}} \leq C\left(\|\rho v \cdot \nabla k\|_{L^{2}}+\|\nabla(\rho v \cdot \nabla k)\|_{L^{2}}\right) \\
& \leq C\left(c_{0}\|v\|_{L^{\infty}}\|\nabla k\|_{L^{2}}+\|\nabla \rho\|_{L^{\infty}}\|v\|_{L^{\infty}}\|\nabla k\|_{L^{2}}\right. \\
&\left.+c_{0}\|\nabla v\|_{L^{4}}\|\nabla k\|_{L^{4}}+c_{0}\|v\|_{L^{\infty}}\left\|\nabla^{2} k\right\|_{L^{2}}\right) \\
& \leq C c_{0}^{\frac{9}{2}} c_{1} c_{2}^{3}, \\
&\left\|G^{\prime}\right\|_{H^{1}} \leq C\left(\|\nabla v\|_{L^{4}}^{2}+\|\nabla v \cdot \rho \cdot \pi\|_{L^{2}}+\left\|\nabla v \cdot \nabla^{2} v\right\|_{L^{2}}+\|\nabla(\nabla v \cdot \rho \cdot \pi)\|_{L^{2}}\right) \\
& \leq C\left(\|\nabla v\|_{L^{4}}^{2}+c_{0}\|\pi\|_{L^{\infty}}\|\nabla v\|_{L^{2}}+\|\nabla v\|_{L^{4}}\left\|\nabla^{2} v\right\|_{L^{4}}+c_{0}\|\pi\|_{L^{\infty}}\left\|\nabla^{2} v\right\|_{L^{2}}\right. \\
&\left.+\|\pi\|_{L^{\infty}}\|\nabla \rho\|_{L^{\infty}}\|\nabla v\|_{L^{2}}+c_{0}\|\nabla v\|_{L^{\infty}}\|\nabla \pi\|_{L^{2}}\right) \\
& \leq C c_{0} c_{1} c_{2}^{2} c_{3} c_{5},
\end{aligned}
$$

and

$$
\|\rho \theta\|_{H^{1}} \leq C\|\rho\|_{H^{3}}\|\theta\|_{H^{1}} \leq C c_{0} c_{1} .
$$

Therefore, inserting (2.65)-(2.68) to (2.64) and integrating the result thus obtained over $(0, t)$, one gets

$$
\int_{0}^{t}\|k\|_{H^{3}}^{2} \mathrm{~d} t \leq C c_{0}^{7}
$$

for $0 \leq t \leq T$.

Combining (2.62), (2.63), and (2.69), we complete the proof of Lemma 2.3.

In the next part, we estimate the viscous dissipation rates of the turbulent flows $\varepsilon$. 
Lemma 2.4 There exists a unique strong solution $\varepsilon$ to the initial boundary value problem (2.6) and (2.9) such that

$$
\begin{aligned}
& \left\|\sqrt{\rho} \varepsilon_{t}\right\|_{L^{2}}^{2}+\|\varepsilon\|_{H^{1}}^{2}+\int_{0}^{t}\left\|\nabla \varepsilon_{t}\right\|_{L^{2}}^{2} \mathrm{~d} s \leq C c_{0}^{5} \\
& \|\varepsilon\|_{H^{2}} \leq C c_{0}^{\frac{9}{2}} c_{1}^{2} c_{2}^{2}
\end{aligned}
$$

for $0 \leq t \leq T$.

Proof We only need to prove the estimates. Differentiating the equation (2.6) with respect to $t$, then multiplying both sides of the result by $\varepsilon_{t}$ and integrating over $\Omega$, one obtains

$$
\begin{aligned}
\frac{1}{2} \frac{\mathrm{d}}{\mathrm{d} t}\left\|\sqrt{\rho} \varepsilon_{t}\right\|_{L^{2}}^{2}+\left\|\nabla \varepsilon_{t}\right\|_{L^{2}}^{2} \\
=-\int \rho_{t} \nu \cdot \nabla \varepsilon \cdot \varepsilon_{t}-\int \rho v_{t} \cdot \nabla \varepsilon \cdot \varepsilon_{t}-2 \int \rho \nu \cdot \nabla \varepsilon_{t} \cdot \varepsilon_{t} \\
\quad+\int\left(\frac{C_{1} G^{\prime} \theta}{\pi}\right)_{t} \cdot \varepsilon_{t}-\int\left(\frac{C_{2} \rho \theta^{2}}{\pi}\right)_{t} \cdot \varepsilon_{t} \\
=\sum_{i=1}^{5} E_{i} .
\end{aligned}
$$

We could evaluate $E_{4}$ and $E_{5}$ in the first place. Because $\pi$ has an upper and a lower bound away from zero, direct calculation yields

$$
\begin{aligned}
E_{4} \leq & C \int\left(\left|G_{t}^{\prime} \theta\right|+\left|G^{\prime} \theta_{t}\right|+\left|G^{\prime} \theta \pi_{t}\right|\right)\left|\varepsilon_{t}\right| \\
\leq & C \int\left(\left|\nabla v_{t} \cdot \nabla v\right|+\left|\rho_{t} \pi \nabla v\right|+\left|\rho \pi_{t} \nabla v\right|+\left|\rho \pi \nabla v_{t}\right|\right)\left|\theta \| \varepsilon_{t}\right| \\
& +C \int\left(|\nabla v|^{2}+|\rho \pi \nabla v|\right)\left|\theta_{t}\left\|\varepsilon_{t}\left|+C \int\left(|\nabla v|^{2}+|\rho \pi \nabla v|\right)\right| \theta\right\| \pi_{t} \| \varepsilon_{t}\right| \\
\leq & C c_{0}^{\frac{1}{2}}\|\theta\|_{L^{\infty}}\|\nabla v\|_{L^{\infty}}\left\|\nabla v_{t}\right\|_{L^{2}}\left\|\sqrt{\rho} \varepsilon_{t}\right\|_{L^{2}}+C c_{0}^{\frac{1}{2}}\|\pi\|_{L^{\infty}}\left\|\sqrt{\rho} \varepsilon_{t}\right\|_{L^{2}}\left\|\rho_{t}\right\|_{L^{6}}\|\nabla v\|_{L^{6}}\|\theta\|_{L^{6}} \\
& +C c_{0}^{\frac{1}{2}}\left\|\sqrt{\rho} \varepsilon_{t}\right\|_{L^{2}}\left\|\pi_{t}\right\|_{L^{6}}\|\nabla v\|_{L^{6}}\|\theta\|_{L^{6}}+C c_{0}\|\pi\|_{L^{\infty}}\|\theta\|_{L^{\infty}}\left\|\nabla v_{t}\right\|_{L^{2}}\left\|\sqrt{\rho} \varepsilon_{t}\right\|_{L^{2}} \\
& +C\left\|\sqrt{\rho} \varepsilon_{t}\right\|_{L^{2}}\left\|\theta_{t}\right\|_{L^{2}}\|\nabla v\|_{L^{\infty}}^{2}+C c_{0}\|\pi\|_{L^{\infty}}\left\|\sqrt{\rho} \varepsilon_{t}\right\|_{L^{2}}\left\|\theta_{t}\right\|_{L^{2}}\|\nabla v\|_{L^{\infty}} \\
& +C c_{0}^{\frac{1}{2}}\left\|\sqrt{\rho} \varepsilon_{t}\right\|_{L^{2}}\left\|\pi_{t}\right\|_{L^{6}}\|\nabla v\|_{L^{6}}^{2}\|\theta\|_{L^{\infty}}+C c_{0}^{\frac{1}{2}}\|\pi\|_{L^{\infty}}\left\|\sqrt{\rho} \varepsilon_{t}\right\|_{L^{2}}\left\|\pi_{t}\right\|_{L^{6}}\|\nabla v\|_{L^{6}}\|\theta\|_{L^{6}} \\
\leq & C \eta^{-1} c_{0} c_{1}^{2} c_{2}^{4} c_{6}^{2} c_{3}^{4} c_{5}^{2}+C c_{0}^{4} c_{1}^{2} c_{2}^{4} c_{5}^{2}+C \eta\left(\left\|\nabla v_{t}\right\|_{L^{2}}^{2}+\left\|\pi_{t}\right\|_{L^{6}}^{2}+\left\|\theta_{t}\right\|_{L^{2}}^{2}\right)\left\|\sqrt{\rho} \varepsilon_{t}\right\|_{L^{2}}^{2} \\
& +C\left\|\sqrt{\rho} \varepsilon_{t}\right\|_{L^{2}}^{2}
\end{aligned}
$$

and

$$
\begin{aligned}
E_{5} & \leq C \int\left|\rho_{t} \theta^{2} \varepsilon_{t}\right|+C \int\left|\theta \theta_{t} \rho \varepsilon_{t}\right|+C \int\left|\rho \theta^{2} \pi_{t} \varepsilon_{t}\right| \\
& \leq C\left\|\rho_{t}\right\|_{L^{3}}\|\theta\|_{L^{4}}^{2}\left\|\varepsilon_{t}\right\|_{L^{6}}+C c_{0}^{\frac{1}{2}}\left\|\sqrt{\rho} \varepsilon_{t}\right\|_{L^{2}}\left\|\theta_{t}\right\|_{L^{2}}\|\theta\|_{L^{\infty}}+C c_{0}^{\frac{1}{2}}\left\|\sqrt{\rho} \varepsilon_{t}\right\|_{L^{2}}\left\|\pi_{t}\right\|_{L^{2}}\|\theta\|_{L^{\infty}}^{2}
\end{aligned}
$$




$$
\begin{aligned}
\leq & C \eta^{-1} c_{0} c_{6}^{4}+C c_{0}^{2} c_{1}^{4} c_{2}^{2}+C\left\|\sqrt{\rho} \varepsilon_{t}\right\|_{L^{2}}^{2}+C \eta\left(\left\|\theta_{t}\right\|_{L^{2}}^{2}+\left\|\pi_{t}\right\|_{L^{2}}^{2}\right)\left\|\sqrt{\rho} \varepsilon_{t}\right\|_{L^{2}}^{2} \\
& +\frac{1}{8}\left\|\nabla \varepsilon_{t}\right\|_{L^{2}}^{2} .
\end{aligned}
$$

Next, using an argument similar to that used in deriving (2.53), (2.54), (2.55), (2.60), and (2.59), respectively, one gets

$$
\begin{aligned}
& E_{1} \leq C c_{0}^{2} c_{2}^{4}\|\nabla \varepsilon\|_{L^{2}}^{2}+C\left\|\sqrt{\rho} \varepsilon_{t}\right\|_{L^{2}}^{2}+\frac{1}{10}\left\|\nabla \varepsilon_{t}\right\|_{L^{2}}^{2}, \\
& E_{2} \leq C \eta^{-1} c_{0}^{2 \gamma+1}\left(\left\|\sqrt{\rho} \varepsilon_{t}\right\|_{L^{2}}^{2}+c_{1}^{2}\|\nabla \varepsilon\|_{L^{2}}^{2}+c_{1}^{4} c_{2}^{4}\right)+\eta\left\|v_{t}\right\|_{H^{1}}^{2}\left\|\sqrt{\rho} \varepsilon_{t}\right\|_{L^{2}}^{2}, \\
& E_{3} \leq C c_{0} c_{2}^{2}\left\|\sqrt{\rho} \varepsilon_{t}\right\|_{L^{2}}^{2}+\frac{1}{10}\left\|\nabla \varepsilon_{t}\right\|_{L^{2}}^{2} \\
& \frac{\mathrm{d}}{\mathrm{d} t}\|\varepsilon\|_{L^{2}}^{2} \leq C\|\varepsilon\|_{L^{2}}^{2}+C c_{0}\left\|\sqrt{\rho} \varepsilon_{t}\right\|_{L^{2}}^{2}
\end{aligned}
$$

and finally

$$
\frac{\mathrm{d}}{\mathrm{d} t}\|\nabla \varepsilon\|_{L^{2}}^{2} \leq \frac{1}{8}\left\|\nabla \varepsilon_{t}\right\|_{L^{2}}^{2}+C\|\nabla \varepsilon\|_{L^{2}}^{2} .
$$

Combining (2.72)-(2.79), one obtains

$$
\begin{aligned}
& \frac{1}{2} \frac{\mathrm{d}}{\mathrm{d} t}\left(\left\|\sqrt{\rho} \varepsilon_{t}\right\|_{L^{2}}^{2}+\|\varepsilon\|_{H^{1}}^{2}\right)+\left\|\nabla \varepsilon_{t}\right\|_{L^{2}}^{2} \\
& \leq C\left(c_{0}^{2} c_{2}^{4}+\eta^{-1} c_{0}^{2 \gamma+1} c_{1}^{2}+\eta\left\|v_{t}\right\|_{H^{1}}^{2}+\eta\left\|\theta_{t}\right\|_{H^{1}}^{2}+\eta\left\|\pi_{t}\right\|_{H^{1}}^{2}\right)\left(\left\|\sqrt{\rho} \varepsilon_{t}\right\|_{L^{2}}^{2}+\|\varepsilon\|_{H^{1}}^{2}\right) \\
& \quad+C \eta^{-1} c_{0} c_{1}^{4} c_{2}^{4} c_{6}^{4} c_{3}^{4} c_{5}^{2}+C c_{0}^{4} c_{1}^{4} c_{2}^{4} c_{5}^{2}
\end{aligned}
$$

setting $\eta=c_{1}^{-1}$ and using the Gronwall inequality, one obtains

$$
\left\|\sqrt{\rho} \varepsilon_{t}\right\|_{L^{2}}^{2}+\|\varepsilon\|_{H^{1}}^{2}+\int_{0}^{t}\left\|\nabla \varepsilon_{t}\right\|_{L^{2}}^{2} \mathrm{~d} s \leq C c_{0}^{5}
$$

for $0 \leq t \leq T$, where we have used the fact that $\lim _{t \rightarrow 0}\left(\left\|\sqrt{\rho} \varepsilon_{t}\right\|_{L^{2}}^{2}+\|\varepsilon\|_{H^{1}}^{2}\right) \leq C c_{0}^{5}$.

Next, applying the standard elliptic regularity result to the equation (2.6) and using (2.81), we have

$$
\begin{aligned}
\|\nabla \varepsilon\|_{H^{1}} \leq & C\left(c_{0}^{\frac{1}{2}}\left\|\sqrt{\rho} \varepsilon_{t}\right\|_{L^{2}}+c_{0}\|v\|_{L^{6}}\|\nabla \varepsilon\|_{L^{3}}+\|\nabla v\|_{L^{6}}^{2}\|\theta\|_{L^{6}}\right. \\
& \left.+c_{0}\|\nabla v\|_{L^{6}}\|\theta\|_{L^{6}}\|\pi\|_{L^{6}}+c_{0}\|\theta\|_{L^{4}}^{2}\right) \\
\leq & C\left(c_{0}^{3} c_{2}^{2} c_{1}^{2}+c_{0} c_{1}\|\nabla \varepsilon\|_{L^{2}}^{\frac{1}{2}}\|\nabla \varepsilon\|_{L^{6}}^{\frac{1}{2}}\right)
\end{aligned}
$$

therefore, by the Young inequality and (2.81), one deduces

$$
\|\varepsilon\|_{H^{2}} \leq C c_{0}^{\frac{9}{2}} c_{1}^{2} c_{2}^{2}
$$

Thus, we complete the proof of Lemma 2.4.

Finally, we estimate the total enthalpy $h$. 
Lemma 2.5 There exists a unique strong solution $h$ to the initial boundary value problem (2.4) and (2.9) such that

$$
\begin{aligned}
& \left\|\sqrt{\rho} h_{t}\right\|_{L^{2}}^{2}+\|h\|_{H^{1}}^{2}+\int_{0}^{t}\left\|\nabla h_{t}\right\|_{L^{2}}^{2} \mathrm{~d} s \leq C c_{0}^{5}, \\
& \|h\|_{H^{2}} \leq C c_{0}^{\frac{7}{2}+\gamma} c_{1}^{2} c_{2}^{2}
\end{aligned}
$$

for $0 \leq t \leq T$.

Proof We only need to prove the estimates. Differentiating equation (2.4) with respect to $t$, multiplying both sides of the result equation by $h_{t}$ and integrating over $\Omega$, one obtains

$$
\begin{aligned}
\frac{\mathrm{d}}{\mathrm{d} t}( & \left.\left\|\sqrt{\rho} h_{t}\right\|_{L^{2}}^{2}+\|h\|_{H^{1}}^{2}\right)+\left\|\nabla h_{t}\right\|_{L^{2}}^{2} \\
= & -\int \rho_{t} v \cdot \nabla h \cdot h_{t}-\int \rho v_{t} \cdot \nabla h \cdot h_{t}-2 \int \rho v \cdot \nabla h_{t} \cdot h_{t}+\int p_{t t} \cdot h_{t} \\
& +\int u_{t} \cdot \nabla p \cdot h_{t}+\int u \cdot \nabla p_{t} \cdot h_{t}+\int S_{k t}^{\prime} \cdot h_{t} \\
= & \sum_{i=1}^{7} H_{i} .
\end{aligned}
$$

First of all, using similar methods of deriving the estimates (2.15), (2.20), and (2.16), respectively, one has

$$
\begin{aligned}
& H_{1} \leq C c_{0}^{2} c_{2}^{4}\|\nabla h\|_{L^{2}}^{2}+C\left\|\sqrt{\rho} h_{t}\right\|_{L^{2}}^{2}+\frac{1}{20}\left\|\nabla h_{t}\right\|_{L^{2}}, \\
& H_{2} \leq C \eta^{-1} c_{0}^{2 \gamma+1}\left(c_{0}^{7} c_{2}^{4}+\left\|\sqrt{\rho} h_{t}\right\|_{L^{2}}^{2}+c_{1}^{2}\|\nabla h\|_{L^{2}}^{2}\right)+\eta\left\|v_{t}\right\|_{H^{1}}^{2}\left\|\sqrt{\rho} h_{t}\right\|_{L^{2}}^{2}, \\
& H_{3} \leq C c_{0} c_{2}^{2}\left\|\sqrt{\rho} h_{t}\right\|_{L^{2}}^{2}+\frac{1}{20}\left\|\nabla h_{t}\right\|_{L^{2}} .
\end{aligned}
$$

Second, differentiating the equation (2.2) with respect to $t$ yields

$$
\rho_{t t}=-\rho_{t} \nabla \cdot v+\rho \nabla \cdot v_{t}+v_{t} \cdot \nabla \rho+v \cdot \nabla \rho_{t} .
$$

Therefore, by direct calculation and using (2.89), we derive

$$
\begin{aligned}
H_{4}= & \int\left[\gamma(\gamma-1) \rho^{\gamma-2} \rho_{t}^{2}-\gamma \rho^{\gamma-1}\left(\rho_{t} \nabla \cdot v+\rho \nabla \cdot v_{t}+v_{t} \cdot \nabla \rho+v \cdot \nabla \rho_{t}\right)\right] \cdot h_{t} \\
\leq & C c_{0}^{\gamma-\frac{3}{2}}\left\|\rho_{t}\right\|_{L^{4}}^{2}\left\|\sqrt{\rho} h_{t}\right\|_{L^{2}}+C c_{0}^{\gamma-\frac{1}{2}}\left\|\rho_{t}\right\|_{L^{3}}\|\nabla v\|_{L^{6}}\left\|\sqrt{\rho} h_{t}\right\|_{L^{2}} \\
& +C c_{0}^{\gamma-\frac{1}{2}}\left\|\sqrt{\rho} h_{t}\right\|_{L^{2}}\left\|\nabla v_{t}\right\|_{L^{2}}+C c_{0}^{\gamma-\frac{1}{2}}\left\|\sqrt{\rho} h_{t}\right\|\left\|_{L^{2}}\right\| v_{t}\left\|_{L^{6}}\right\| \nabla \rho \|_{L^{3}} \\
& +C c_{0}^{\gamma-\frac{1}{2}}\left\|\nabla \rho_{t}\right\|_{L^{2}}\|v\|_{L^{\infty}}\left\|\sqrt{\rho} h_{t}\right\|_{L^{2}} \\
\leq & C\left(c_{0}^{2 \gamma+1} c_{2}^{4}+\eta^{-1} c_{0}^{2 \gamma}+\left\|\sqrt{\rho} h_{t}\right\|_{L^{2}}^{2}+\eta\left\|v_{t}\right\|_{H^{1}}^{2}\left\|\sqrt{\rho} h_{t}\right\|_{L^{2}}^{2}\right)+\frac{1}{20}\left\|\nabla h_{t}\right\|_{L^{2}}^{2} .
\end{aligned}
$$


Third, simple calculation and (2.26) lead to

$$
\begin{aligned}
H_{5} & \leq C c_{0}^{\gamma-\frac{1}{2}}\left\|\sqrt{\rho} h_{t}\right\|_{L^{2}}\|\nabla \rho\|_{L^{3}}\left\|u_{t}\right\|_{L^{6}} \leq C c_{0}^{\gamma-\frac{1}{2}}\left\|\sqrt{\rho} h_{t}\right\|_{L^{2}}\|\nabla \rho\|_{L^{3}}\left(\left\|u_{t}\right\|_{L^{2}}+\left\|\nabla u_{t}\right\|_{L^{2}}\right) \\
& \leq C c_{0}^{2 \gamma+1}\left\|\sqrt{\rho} h_{t}\right\|_{L^{2}}^{2}+C c_{0}^{2 \gamma+5}+C\left\|\nabla u_{t}\right\|_{L^{2}}^{2} .
\end{aligned}
$$

Next, by direct calculation, we know that $\nabla p_{t}=\gamma(\gamma-1) \rho^{\gamma-2} \rho_{t} \nabla \rho+\gamma \rho^{\gamma-1} \nabla \rho_{t}$. Therefore,

$$
\begin{aligned}
H_{6} \leq & C c_{0}^{\gamma-2} \int\left|\rho_{t}\|u\| \nabla \rho\left\|h_{t}\left|+C c_{0}^{\gamma-1} \int\right| u\right\| \nabla \rho_{t} \| h_{t}\right| \\
\leq & C c_{0}^{\gamma-2}\|\nabla \rho\|_{L^{\infty}}\left\|\rho_{t}\right\|_{L^{3}}\|u\|_{L^{2}}\left(\left\|\sqrt{\rho} h_{t}\right\|_{L^{2}}+\left\|\nabla h_{t}\right\|_{L^{2}}\right) \\
& +C c_{0}^{\gamma-1}\|u\|_{L^{3}}\left\|\nabla \rho_{t}\right\|_{L^{2}}\left(\left\|\sqrt{\rho} h_{t}\right\|_{L^{2}}+\left\|\nabla h_{t}\right\|_{L^{2}}\right) \\
\leq & C c_{0}^{7+2 \gamma} c_{2}^{2}+C\left\|\sqrt{\rho} h_{t}\right\|_{L^{2}}^{2}+\frac{1}{20}\left\|\nabla h_{t}\right\|_{L^{2}}^{2} .
\end{aligned}
$$

Last, simple calculation yields $\left|S_{k t}^{\prime}\right| \leq C|\nabla v|\left|\nabla v_{t}\right|+C \rho^{\gamma-1}\left|\rho_{t}\right||\nabla \rho|^{2}+C \rho^{\gamma-1}\left|\nabla \rho_{t}\right||\nabla \rho|$, thus

$$
\begin{aligned}
H_{7} \leq & C \int\left|\nabla v_{t}\right||\nabla v|\left|h_{t}\right|+C c_{0}^{\gamma-1} \int\left|\rho_{t}\right||\nabla \rho|^{2}\left|h_{t}\right|+C c_{0}^{\gamma-1} \int\left|\nabla \rho_{t}\|\nabla \rho\| h_{t}\right| \\
\leq & C c_{0}^{\frac{1}{2}}\|\nabla v\|_{L^{\infty}}\left\|\nabla v_{t}\right\|_{L^{2}}\left\|\sqrt{\rho} h_{t}\right\|_{L^{2}}+C c_{0}^{\gamma-\frac{1}{2}}\left\|\rho_{t}\right\|_{L^{6}}\|\nabla \rho\|_{L^{6}}^{2}\left\|\sqrt{\rho} h_{t}\right\|_{L^{2}} \\
& +C c_{0}^{\gamma-\frac{1}{2}}\|\nabla \rho\|_{L^{\infty}}\left\|\nabla \rho_{t}\right\|_{L^{2}}\left\|\sqrt{\rho} h_{t}\right\|_{L^{2}} \\
\leq & C\left(\eta^{-1} c_{0} c_{3}^{2}+c_{0}^{5+2 \gamma} c_{2}^{2}+\eta\left\|\nabla v_{t}\right\|_{L^{2}}^{2}\left\|\sqrt{\rho} h_{t}\right\|_{L^{2}}^{2}+\left\|\sqrt{\rho} h_{t}\right\|_{L^{2}}^{2}\right) .
\end{aligned}
$$

Furthermore, we easily have

$$
\frac{\mathrm{d}}{\mathrm{d} t}\|h\|_{L^{2}}^{2} \leq C c_{0}\left\|\sqrt{\rho} h_{t}\right\|_{L^{2}}^{2}+C\|h\|_{L^{2}}^{2}
$$

and

$$
\frac{\mathrm{d}}{\mathrm{d} t}\|\nabla h\|_{L^{2}}^{2} \leq C\|\nabla h\|_{L^{2}}^{2}+\frac{1}{10}\left\|\nabla h_{t}\right\|_{L^{2}}^{2} .
$$

Consequently, combining (2.85)-(2.95), one deduces

$$
\begin{aligned}
\frac{\mathrm{d}}{\mathrm{d} t}( & \left.\left\|\sqrt{\rho} h_{t}\right\|_{L^{2}}^{2}+\|h\|_{H^{1}}^{2}\right)+\left\|\nabla h_{t}\right\|_{L^{2}}^{2} \\
\leq & C\left(c_{0}^{2 \gamma+1} c_{2}^{4}+\eta^{-1} c_{0}^{2 \gamma+1} c_{1}^{2}+\eta\left\|v_{t}\right\|_{H^{1}}^{2}\right)\left(\left\|\sqrt{\rho} h_{t}\right\|_{L^{2}}^{2}+\|h\|_{H^{1}}^{2}\right) \\
& +C\left(c_{0}^{7+2 \gamma} c_{2}^{4}+\eta^{-1} c_{0}^{8+2 \gamma} c_{2}^{4} c_{3}^{2}\right),
\end{aligned}
$$

setting $\eta=c_{1}^{-1}$ and using the Gronwall inequality, we get

$$
\left\|\sqrt{\rho} h_{t}\right\|_{L^{2}}^{2}+\|h\|_{H^{1}}^{2}+\int_{0}^{t}\left\|\nabla h_{t}\right\|_{L^{2}}^{2} \mathrm{~d} s \leq C c_{0}^{5}
$$

for $0 \leq t \leq T$, where we have used the fact that $\lim _{t \rightarrow 0}\left(\left\|\sqrt{\rho} h_{t}\right\|_{L^{2}}^{2}+\|h\|_{H^{1}}^{2}\right) \leq C c_{0}^{5}$. 
Next, using (2.97) and the standard elliptic regularity result of the equation (2.4), one obtains

$$
\begin{aligned}
\|\nabla h\|_{H^{1}} \leq & C\left(c_{0}^{\frac{1}{2}}\left\|\sqrt{\rho} h_{t}\right\|_{L^{2}}+c_{0}\|v\|_{L^{6}}\|\nabla h\|_{L^{3}}+c_{0}^{\gamma-1}\left\|\rho_{t}\right\|_{L^{2}}+c_{0}^{\gamma-1}\|u\|_{L^{6}}\|\nabla \rho\|_{L^{3}}\right. \\
& \left.+\|\nabla v\|_{L^{4}}^{2}+c_{0}^{\gamma-1}\|\nabla \rho\|_{L^{4}}^{2}\right) \\
\leq & C c_{0}^{\frac{5}{2}+\gamma} c_{2}^{2}+C c_{0} c_{1}\|\nabla h\|_{L^{2}}^{\frac{1}{2}}\|\nabla h\|_{H^{1}}^{\frac{1}{2}},
\end{aligned}
$$

then the Young inequality and (2.97) yield

$$
\|h\|_{H^{2}} \leq C c_{0}^{\frac{7}{2}+\gamma} c_{1}^{2} c_{2}^{2}
$$

Thus, we have finished the proof of Lemma 2.5.

Next, let us define $c_{i}(i=1, \ldots, 6)$ as follows:

$$
\begin{aligned}
& c_{1}=C c_{0}^{7+2 \gamma}, \quad c_{2}=C c_{0}^{\frac{5}{2}+3 \gamma} c_{1}^{2}, \quad c_{5}=C c_{0}^{\frac{7}{2}} c_{1} c_{2}^{2}, \\
& c_{6}=C c_{0}^{\frac{9}{2}} c_{1}^{2} c_{2}^{2}, \quad c_{3}=C c_{0}^{\frac{13}{2}+3 \gamma} c_{1}^{4} c_{2} c_{5}, \quad c_{4}=C c_{0}^{9+6 \gamma} c_{1}^{5} c_{2}^{2},
\end{aligned}
$$

then we conclude from Lemma 2.1 to Lemma 2.5 that

$$
\left\{\begin{array}{l}
\sup _{0 \leq t \leq T}\left(\|u\|_{H^{1}}+\|k\|_{H^{1}}+\|\varepsilon\|_{H^{1}}\right) \\
\quad+\int_{0}^{T}\left(\|k\|_{H^{3}}^{2}+\left\|u_{t}\right\|_{H^{1}}^{2}+\left\|k_{t}\right\|_{H^{1}}^{2}+\left\|\varepsilon_{t}\right\|_{H^{1}}^{2}\right) \mathrm{d} t \leq c_{1}, \\
\sup _{0 \leq t \leq T}\|u\|_{H^{2}} \leq c_{2}, \quad \sup _{0 \leq t \leq T}\|u\|_{H^{3}} \leq c_{3}, \quad \int_{0}^{T}\|u\|_{H^{4}}^{2} \mathrm{~d} t \leq c_{4}, \\
\sup _{0 \leq t \leq T}\|k\|_{H^{2}} \leq c_{5}, \quad \sup _{0 \leq t \leq T}\|\varepsilon\|_{H^{2}} \leq c_{6},
\end{array}\right.
$$

and

$$
\left\{\begin{array}{l}
\|\rho\|_{H^{3}(\Omega)} \leq C c_{0}, \quad\left\|\rho_{t}\right\|_{H^{1}(\Omega)} \leq C c_{0} c_{2} \\
\left\|\sqrt{\rho} h_{t}\right\|_{L^{2}}^{2}+\|h\|_{H^{1}}^{2}+\int_{0}^{t}\left\|\nabla h_{t}\right\|_{L^{2}}^{2} \mathrm{~d} s \leq C c_{0}^{5} \\
\|h\|_{H^{2}} \leq C c_{0}^{\frac{7}{2}+\gamma} c_{1}^{2} c_{2}^{2}
\end{array}\right.
$$

for $0 \leq t \leq T$.

Using a standard proof as that in [13], we complete the proof of Theorem 2.1.

\section{Existence of strong solutions to the $\boldsymbol{k}-\varepsilon$ equations}

Theorem 3.1 There exist a small time $T^{*}>0$ and a unique strong solution $(\rho, u, h, k, \varepsilon)$ to the initial boundary value problem (1.1)-(1.10) such that

$$
\begin{array}{ll}
\rho \in C\left(0, T^{*} ; H^{3}\right), & \rho_{t} \in C\left(0, T^{*} ; H^{1}\right), \quad u \in C\left(0, T^{*} ; H^{3}\right) \cap L^{2}\left(0, T^{*} ; H^{4}\right), \\
u_{t} \in L^{2}\left(0, T^{*} ; H^{1}\right), & k \in C\left(0, T^{*} ; H^{2}\right) \cap L^{2}\left(0, T^{*} ; H^{3}\right), \\
k_{t} \in L^{2}\left(0, T^{*} ; H^{1}\right), & \varepsilon \in C\left(0, T^{*} ; H^{2}\right), \quad \varepsilon_{t} \in L^{2}\left(0, T^{*} ; H^{1}\right), \\
h \in C\left(0, T^{*} ; H^{2}\right), & h_{t} \in L^{2}\left(0, T^{*} ; H^{1}\right), \\
\left(\sqrt{\rho} u_{t}, \sqrt{\rho} k_{t}, \sqrt{\rho} \varepsilon_{t}, \sqrt{\rho} h_{t}\right) \in L^{\infty}\left(0, T^{*} ; L^{2}\right) .
\end{array}
$$


Proof Our proof will be based on the iteration argument and on the results in the last section (especially Theorem 2.1).

First, using the regularity effect of the classical heat equation, we can construct functions $\left(u^{0}=u^{0}(x, t), k^{0}=k^{0}(x, t), \varepsilon^{0}=\varepsilon^{0}(x, t)\right)$ satisfying $\left(u^{0}(x, 0), k^{0}(x, 0), \varepsilon^{0}(x, 0)\right)=\left(u_{0}(x), k_{0}(x)\right.$, $\left.\varepsilon_{0}(x)\right)$ and

$$
\left\{\begin{array}{l}
\sup _{0 \leq t \leq T}\left(\left\|u^{0}\right\|_{H^{1}}+\left\|k^{0}\right\|_{H^{1}}+\left\|\varepsilon^{0}\right\|_{H^{1}}\right) \\
\quad+\int_{0}^{T}\left(\left\|k^{0}\right\|_{H^{3}}^{2}+\left\|u_{t}^{0}\right\|_{H^{1}}^{2}+\left\|k_{t}^{0}\right\|_{H^{1}}^{2}+\left\|\varepsilon_{t}^{0}\right\|_{H^{1}}^{2}\right) \mathrm{d} t \leq c_{1}, \\
\sup _{0 \leq t \leq T}\left\|u^{0}\right\|_{H^{2}} \leq c_{2}, \quad \sup _{0 \leq t \leq T}\left\|u^{0}\right\|_{H^{3}} \leq c_{3}, \quad \int_{0}^{T}\left\|u^{0}\right\|_{H^{4}}^{2} \mathrm{~d} t \leq c_{4}, \\
\sup _{0 \leq t \leq T}\left\|k^{0}\right\|_{H^{2}} \leq c_{5}, \quad \sup _{0 \leq t \leq T}\left\|\varepsilon^{0}\right\|_{H^{2}} \leq c_{6} .
\end{array}\right.
$$

Therefore it follows from Theorem 2.1 that there exists a unique strong solution $\left(\rho^{1}, u^{1}\right.$, $\left.h^{1}, k^{1}, \varepsilon^{1}\right)$ to the linearized problem (2.2)-(2.6) with $v, \pi, \theta$ replaced by $u^{0}, k^{0}, \varepsilon^{0}$, respectively, which satisfies the regularity estimates (2.99) and (2.100). Similarly, we construct approximate solutions $\left(\rho^{n}, u^{n}, h^{n}, k^{n}, \varepsilon^{n}\right)$, inductively, as follows: assuming that $u^{n-1}, k^{n-1}$, $\varepsilon^{n-1}$ have been defined for $n \geq 1$, let $\left(\rho^{n}, u^{n}, h^{n}, k^{n}, \varepsilon^{n}\right)$ be the unique solution to the linearized problem (2.2)-(2.6) with $v, \pi, \theta$ replaced by $u^{n-1}, k^{n-1}, \varepsilon^{n-1}$, respectively. Then it follows from Theorem 2.1 that there exists a constant $\widetilde{C}>1$ such that

$$
\begin{aligned}
& \sup _{0 \leq t \leq T}\left(\left\|\rho^{n}\right\|_{H^{3}}+\left\|\rho_{t}^{n}\right\|_{H^{1}}\right)+\sup _{0 \leq t \leq T}\left(\left\|u^{n}\right\|_{H^{3}}+\left\|k^{n}\right\|_{H^{2}}+\left\|\varepsilon^{n}\right\|_{H^{2}}+\left\|h^{n}\right\|_{H^{2}}\right) \\
& \quad+\sup _{0 \leq t \leq T}\left(\left\|\sqrt{\rho^{n}} u_{t}^{n}\right\|_{L^{2}}+\left\|\sqrt{\rho^{n}} h_{t}^{n}\right\|_{L^{2}}+\left\|\sqrt{\rho^{n}} k_{t}^{n}\right\|_{L^{2}}+\left\|\sqrt{\rho^{n}} \varepsilon_{t}^{n}\right\|_{L^{2}}\right) \\
& \quad+\int_{0}^{T}\left(\left\|u_{t}^{n}\right\|_{H^{1}}^{2}+\left\|h_{t}^{n}\right\|_{H^{1}}^{2}+\left\|k_{t}^{n}\right\|_{H^{1}}^{2}+\left\|\varepsilon_{t}^{n}\right\|_{H^{1}}^{2}+\left\|u^{n}\right\|_{H^{4}}^{2}+\left\|k^{n}\right\|_{H^{3}}^{2}\right) \leq \widetilde{C}
\end{aligned}
$$

for all $n \geq 1$. Throughout the proof, we denote by $\widetilde{C}$ a generic constant depending only on $m, M, \gamma,|\Omega|$, and $c_{0}$, but independent of $n$. Next, we will show that the full sequence $\left(\rho^{n}, u^{n}, h^{n}, k^{n}, \varepsilon^{n}\right)$ converges to a solution to the original nonlinear problem (1.1)-(1.10) in a strong sense.

Define $\bar{\rho}^{n+1}=\rho^{n+1}-\rho^{n}, \bar{u}^{n+1}=u^{n+1}-u^{n}, \bar{h}^{n+1}=h^{n+1}-h^{n}, \bar{k}^{n+1}=k^{n+1}-k^{n}, \bar{\varepsilon}^{n+1}=\varepsilon^{n+1}-\varepsilon^{n}$, $\bar{p}^{n+1}=p^{n+1}-p^{n}=\left(\rho^{n+1}\right)^{\gamma}-\left(\rho^{n}\right)^{\gamma}$.

Then, by equations (2.2)-(2.6), we deduce that $\left(\bar{\rho}^{n+1}, \bar{u}^{n+1}, \bar{h}^{n+1}, \bar{k}^{n+1}, \bar{\varepsilon}^{n+1}, \bar{p}^{n+1}\right)$ satisfy the following equations:

$$
\begin{aligned}
& \bar{\rho}_{t}^{n+1}+\nabla \cdot\left(\bar{\rho}^{n+1} u^{n}+\rho^{n} \bar{u}^{n}\right)=0, \\
& \rho^{n+1} \bar{u}_{t}^{n+1}+\bar{\rho}^{n+1} u_{t}^{n}+\rho^{n+1} u^{n} \cdot \nabla \bar{u}^{n+1}+\bar{\rho}^{n+1} u^{n} \cdot \nabla u^{n}+\rho^{n} \bar{u}^{n} \cdot \nabla u^{n} \\
& \quad-\Delta \bar{u}^{n+1}-\nabla\left(\nabla \cdot \bar{u}^{n+1}\right)+\nabla \bar{p}^{n+1}=\frac{-2}{3} \nabla\left(\bar{\rho}^{n+1} k^{n}+\rho^{n} \bar{k}^{n}\right), \\
& \rho^{n+1} \bar{h}_{t}^{n+1}+\bar{\rho}^{n+1} h_{t}^{n}+\rho^{n+1} u^{n} \cdot \nabla \bar{h}^{n+1}+\bar{\rho}^{n+1} u^{n} \cdot \nabla h^{n}+\rho^{n} \bar{u}^{n} \cdot \nabla h^{n}-\Delta \bar{h}^{n+1} \\
& \quad=\bar{p}_{t}^{n+1}+\bar{u}^{n+1} \cdot \nabla p^{n+1}+u^{n} \cdot \nabla \bar{p}^{n+1}+S_{k, n+1}^{\prime}-S_{k, n}^{\prime}, \\
& \rho^{n+1} \bar{k}_{t}^{n+1}+\bar{\rho}^{n+1} k_{t}^{n}+\rho^{n+1} u^{n} \cdot \nabla \bar{k}^{n+1}+\bar{\rho}^{n+1} u^{n} \cdot \nabla k^{n}+\rho^{n} \bar{u}^{n} \cdot \nabla k^{n}-\Delta \bar{k}^{n+1} \\
& \quad=G_{n+1}^{\prime}-G_{n}^{\prime}-\left(\rho^{n+1} \varepsilon^{n}-\rho^{n} \varepsilon^{n-1}\right),
\end{aligned}
$$




$$
\begin{aligned}
& \rho^{n+1} \bar{\varepsilon}_{t}^{n+1}+\bar{\rho}^{n+1} \varepsilon_{t}^{n}+\rho^{n+1} u^{n} \cdot \nabla \bar{\varepsilon}^{n+1}+\bar{\rho}^{n+1} u^{n} \cdot \nabla \varepsilon^{n}+\rho^{n} \bar{u}^{n} \cdot \nabla \varepsilon^{n}-\Delta \bar{\varepsilon}^{n+1} \\
& =C_{1}\left(\frac{G_{n+1}^{\prime} \varepsilon^{n}}{k^{n}}-\frac{G_{n}^{\prime} \varepsilon^{n-1}}{k^{n-1}}\right)-C_{2}\left(\frac{\rho^{n+1}\left(\varepsilon^{n}\right)^{2}}{k^{n}}-\frac{\rho^{n}\left(\varepsilon^{n-1}\right)^{2}}{k^{n-1}}\right),
\end{aligned}
$$

where

$$
\begin{aligned}
& S_{k, n+1}^{\prime}=\left[\mu\left(\partial_{j} u_{i}^{n}+\partial_{i} u_{j}^{n}\right)-\frac{2}{3} \delta_{i j} \mu \partial_{k} u_{k}^{n}\right] \partial_{j} u_{i}^{n}+\frac{\mu_{t}}{\left(\rho^{n+1}\right)^{2}} \partial_{j} p^{n+1} \partial_{j} \rho^{n+1}, \\
& G_{n+1}^{\prime}=\partial_{j} u_{i}^{n}\left[\mu_{e}\left(\partial_{j} u_{i}^{n}+\partial_{i} u_{j}^{n}\right)-\frac{2}{3} \delta_{i j}\left(\rho^{n+1} k^{n}+\mu_{e} \partial_{l} u_{l}^{n}\right)\right] .
\end{aligned}
$$

To evaluate $\left\|\bar{\rho}^{n+1}\right\|_{L^{2}}$, multiplying both sides of the equation (3.3) by $\bar{\rho}^{n+1}$ and integrating the result over $\Omega$, we get

$$
\begin{aligned}
& \frac{1}{2} \frac{\mathrm{d}}{\mathrm{d} t}\left\|\bar{\rho}^{n+1}\right\|_{L^{2}}^{2} \\
& \quad=-\int \nabla \cdot\left(\bar{\rho}^{n+1} u^{n}+\rho^{n} \bar{u}^{n}\right) \cdot \bar{\rho}^{n+1} \\
& \quad=-\int\left(\bar{\rho}^{n+1}\right)^{2} \nabla \cdot u^{n}+\bar{\rho}^{n+1} u^{n} \cdot \nabla \bar{\rho}^{n+1}+\rho^{n} \bar{\rho}^{n+1} \nabla \cdot \bar{u}^{n}+\bar{\rho}^{n+1} \bar{u}^{n} \cdot \nabla \rho^{n} .
\end{aligned}
$$

Applying integration by parts to the second term of the second equality of (3.10) and using the Hölder, Sobolev, and Young inequalities yield

$$
\begin{aligned}
\frac{\mathrm{d}}{\mathrm{d} t}\left\|\bar{\rho}^{n+1}\right\|_{L^{2}}^{2} & \leq C\left(\left\|\nabla u^{n}\right\|_{L^{\infty}}\left\|\bar{\rho}^{n+1}\right\|_{L^{2}}^{2}+\left\|\nabla \bar{u}^{n}\right\|_{L^{2}}\left\|\bar{\rho}^{n+1}\right\|_{L^{2}}+\left\|\bar{u}^{n}\right\|_{L^{6}}\left\|\nabla \rho^{n}\right\|_{L^{3}}\left\|\bar{\rho}^{n+1}\right\|_{L^{2}}\right) \\
& \leq \widetilde{C}\left(1+\eta^{-1}\right)\left\|\bar{\rho}^{n+1}\right\|_{L^{2}}^{2}+\widetilde{C} \eta\left\|\nabla \bar{u}^{n}\right\|_{H^{1}}^{2},
\end{aligned}
$$

where (3.2) has been used and $0<\eta<1$ is a small constant to be determined later.

Next, multiplying both sides of (3.4) by $\bar{u}^{n+1}$ and integrating the result thus derived over $\Omega$, one obtains

$$
\begin{aligned}
& \frac{1}{2} \frac{\mathrm{d}}{\mathrm{d} t}\left\|\sqrt{\rho^{n+1}} \bar{u}^{n+1}\right\|_{L^{2}}^{2}+\left\|\nabla \bar{u}^{n+1}\right\|_{L^{2}}^{2}+\left\|\nabla \cdot \bar{u}^{n+1}\right\|_{L^{2}}^{2} \\
& =-\int \bar{\rho}^{n+1} u_{t}^{n} \cdot \bar{u}^{n+1}-\int \bar{\rho}^{n+1} u^{n} \cdot \nabla u^{n} \cdot \bar{u}^{n+1}-\int \rho^{n} \bar{u}^{n} \cdot \nabla u^{n} \cdot \bar{u}^{n+1}-\int \nabla \bar{p}^{n+1} \cdot \bar{u}^{n+1} \\
& \quad+\int \frac{-2}{3} \nabla\left(\bar{\rho}^{n+1} k^{n}+\rho^{n} \bar{k}^{n+1}\right) \cdot \bar{u}^{n+1} \\
& =\sum_{i=1}^{5} L_{i} .
\end{aligned}
$$

Using the Hölder, Sobolev, and Young inequalities and (3.2), we estimate $L_{1}, L_{2}$, and $L_{3}$, respectively, as follows:

$$
\begin{aligned}
L_{1} & \leq C\left\|\bar{\rho}^{n+1}\right\|_{L^{2}}\left\|u_{t}^{n}\right\|_{L^{3}}\left\|\bar{u}^{n+1}\right\|_{L^{6}} \leq C\left\|\bar{\rho}^{n+1}\right\|_{L^{2}}\left\|u_{t}^{n}\right\|_{L^{3}}\left(\left\|\sqrt{\rho^{n+1}} \bar{u}^{n+1}\right\|_{L^{2}}+\left\|\nabla \bar{u}^{n+1}\right\|_{L^{2}}\right) \\
& \leq \widetilde{C}\left\|u_{t}^{n}\right\|_{L^{3}}^{2}\left\|\bar{\rho}^{n+1}\right\|_{L^{2}}^{2}+\widetilde{C}\left\|\sqrt{\rho^{n+1}} \bar{u}^{n+1}\right\|_{L^{2}}^{2}+\frac{1}{8}\left\|\nabla \bar{u}^{n+1}\right\|_{L^{2}}^{2},
\end{aligned}
$$




$$
\begin{aligned}
L_{2} & \leq C\left\|\bar{\rho}^{n+1}\right\|_{L^{2}}\left\|u^{n}\right\|_{L^{6}}\left\|\nabla u^{n}\right\|_{L^{6}}\left\|\bar{u}^{n+1}\right\|_{L^{6}} \\
& \leq \widetilde{C}\left\|\bar{\rho}^{n+1}\right\|_{L^{2}}^{2}+\widetilde{C}\left\|\sqrt{\rho^{n+1}} \bar{u}^{n+1}\right\|_{L^{2}}^{2}+\frac{1}{8}\left\|\nabla \bar{u}^{n+1}\right\|_{L^{2}}^{2}, \\
L_{3} & \leq C\left\|\bar{u}^{n}\right\|_{L^{6}}\left\|\nabla u^{n}\right\|_{L^{3}}\left\|\sqrt{\rho^{n+1}} \bar{u}^{n+1}\right\|_{L^{2}} \leq \widetilde{C} \eta^{-1}\left\|\sqrt{\rho^{n+1}} \bar{u}^{n+1}\right\|_{L^{2}}^{2}+\eta\left\|\bar{u}^{n}\right\|_{H^{1}}^{2} .
\end{aligned}
$$

Then one deduces by integration by parts that

$$
L_{4}=\int \bar{p}^{n+1} \nabla \cdot \bar{u}^{n+1} \leq C \int \bar{\rho}^{n+1} \nabla \cdot \bar{u}^{n+1} \leq \widetilde{C}\left\|\bar{\rho}^{n+1}\right\|_{L^{2}}^{2}+\frac{1}{8}\left\|\nabla \bar{u}^{n+1}\right\|_{L^{2}}^{2}
$$

and

$$
\begin{aligned}
L_{5}= & \frac{2}{3} \int \bar{\rho}^{n+1} k^{n} \nabla \cdot \bar{u}^{n+1}-\bar{k}^{n} \nabla \rho^{n} \cdot \bar{u}^{n+1}-\rho^{n} \nabla \bar{k}^{n} \cdot \bar{u}^{n+1} \\
\leq & C\left\|\bar{\rho}^{n+1}\right\|_{L^{2}}\left\|\nabla \bar{u}^{n+1}\right\|_{L^{2}}+C\left\|\bar{k}^{n}\right\|_{L^{6}}\left\|\nabla \rho^{n}\right\|_{L^{3}}\left\|\sqrt{\rho^{n+1}} \bar{u}^{n+1}\right\|_{L^{2}} \\
& +C\left\|\nabla \bar{k}^{n}\right\|_{L^{2}}\left\|\sqrt{\rho^{n+1}} \bar{u}^{n+1}\right\|_{L^{2}} \\
\leq & \widetilde{C}\left(1+\eta^{-1}\right)\left(\left\|\bar{\rho}^{n+1}\right\|_{L^{2}}^{2}+\left\|\sqrt{\rho^{n+1}} \bar{u}^{n+1}\right\|_{L^{2}}^{2}\right)+\frac{1}{8}\left\|\nabla \bar{u}^{n+1}\right\|_{L^{2}}^{2}+\widetilde{C} \eta\left\|\bar{k}^{n}\right\|_{H^{1}}^{2} .
\end{aligned}
$$

Inserting (3.13)-(3.17) to (3.12) and using inequality $\left\|\bar{u}^{n+1}\right\|_{L^{2}} \leq \widetilde{C}\left\|\sqrt{\rho^{n+1}} \bar{u}^{n+1}\right\|_{L^{2}}$, one has

$$
\begin{gathered}
\frac{\mathrm{d}}{\mathrm{d} t}\left\|\sqrt{\rho^{n+1}} \bar{u}^{n+1}\right\|_{L^{2}}^{2}+\left\|\bar{u}^{n+1}\right\|_{H^{1}}^{2} \leq \\
\widetilde{C}\left(1+\eta^{-1}+\left\|u_{t}^{n}\right\|_{L^{3}}^{2}\right)\left(\left\|\bar{\rho}^{n+1}\right\|_{L^{2}}^{2}+\left\|\sqrt{\rho^{n+1}} \bar{u}^{n+1}\right\|_{L^{2}}^{2}\right) \\
+\widetilde{C} \eta\left\|\bar{k}^{n}\right\|_{H^{1}}^{2}+\widetilde{C} \eta\left\|\bar{u}^{n}\right\|_{H^{1}}^{2} .
\end{gathered}
$$

Then, multiplying both sides of (3.5) by $\bar{h}^{n+1}$ and integrating the result thus got over $\Omega$, one obtains

$$
\begin{aligned}
\frac{1}{2} & \frac{\mathrm{d}}{\mathrm{d} t}\left\|\sqrt{\rho^{n+1}} \bar{h}^{n+1}\right\|_{L^{2}}^{2}+\left\|\nabla \bar{h}^{n+1}\right\|_{L^{2}}^{2} \\
= & -\int \bar{\rho}^{n+1} h_{t}^{n} \cdot \bar{h}^{n+1}-\int \bar{\rho}^{n+1} u^{n} \cdot \nabla h^{n} \cdot \bar{h}^{n+1}-\int \rho^{n} \bar{u}^{n} \cdot \nabla h^{n} \cdot \bar{h}^{n+1} \\
& +\int\left(\bar{p}_{t}^{n+1}+\bar{u}^{n+1} \cdot \nabla p^{n+1}+u^{n} \cdot \nabla \bar{p}^{n+1}\right) \cdot \bar{h}^{n+1}+\int\left(S_{k, n+1}^{\prime}-S_{k, n}^{\prime}\right) \cdot \bar{h}^{n+1} \\
= & \sum_{i=1}^{5} M_{i} .
\end{aligned}
$$

First, using similar methods of deriving (3.13), (3.14), and (3.15), respectively, one easily obtains

$$
\begin{aligned}
& M_{1} \leq \widetilde{C}\left\|h_{t}^{n}\right\|_{L^{3}}^{2}\left\|\bar{\rho}^{n+1}\right\|_{L^{2}}^{2}+\widetilde{C}\left\|\sqrt{\rho^{n+1}} \bar{h}^{n+1}\right\|_{L^{2}}^{2}+\frac{1}{20}\left\|\nabla \bar{h}^{n+1}\right\|_{L^{2}}^{2}, \\
& M_{2} \leq \widetilde{C}\left\|\bar{\rho}^{n+1}\right\|_{L^{2}}^{2}+\widetilde{C}\left\|\sqrt{\rho^{n+1}} \bar{h}^{n+1}\right\|_{L^{2}}^{2}+\frac{1}{20}\left\|\nabla \bar{h}^{n+1}\right\|_{L^{2}}^{2}, \\
& M_{3} \leq \widetilde{C} \eta^{-1}\left\|\sqrt{\rho^{n+1}} \bar{h}^{n+1}\right\|_{L^{2}}^{2}+\eta\left\|\bar{u}^{n}\right\|_{H^{1}}^{2} .
\end{aligned}
$$


Second, simple calculation leads to

$$
\begin{aligned}
M_{4}= & \int\left[\gamma\left(\rho^{n+1}\right)^{\gamma-1} \rho_{t}^{n+1}-\gamma\left(\rho^{n}\right)^{\gamma-1} \rho_{t}^{n}\right] \cdot \bar{h}^{n+1}+\int \bar{u}^{n+1} \cdot \nabla p^{n+1} \bar{h}^{n+1} \\
& +\int u^{n} \cdot \nabla \bar{p}^{n+1} \bar{h}^{n+1} .
\end{aligned}
$$

By the differential mean value theorem, the first integral of (3.23) can be controlled as

$$
\begin{aligned}
& \int\left[\gamma\left(\rho^{n+1}\right)^{\gamma-1} \rho_{t}^{n+1}-\gamma\left(\rho^{n}\right)^{\gamma-1} \rho_{t}^{n}\right] \cdot \bar{h}^{n+1} \\
& \leq C \int\left|\bar{\rho}^{n+1}\right|\left|\rho_{t}^{n+1}\right|\left|\bar{h}^{n+1}\right|+\int \gamma\left(\rho^{n}\right)^{\gamma-1} \bar{\rho}_{t}^{n+1} \cdot \bar{h}^{n+1} .
\end{aligned}
$$

By the equation (3.3), the second integral on the right-hand side of (3.24) can be estimated as

$$
\begin{aligned}
& \int \gamma\left(\rho^{n}\right)^{\gamma-1} \bar{\rho}_{t}^{n+1} \cdot \bar{h}^{n+1} \\
& =-\int \gamma\left(\rho^{n}\right)^{\gamma-1} \nabla \cdot\left(\bar{\rho}^{n+1} u^{n}+\rho^{n} \bar{u}^{n}\right) \cdot \bar{h}^{n+1} \\
& \leq C \int\left|\nabla \rho^{n}\right|\left|\bar{h}^{n+1}\right|\left|\bar{\rho}^{n+1}\right|\left|u^{n}\right|+C \int\left|\bar{\rho}^{n+1}\right|\left|u^{n}\right|\left|\nabla \bar{h}^{n+1}\right| \\
& \quad+C \int\left(\left|\nabla \rho^{n}\right|\left|\bar{u}^{n}\right|+\left|\rho^{n}\right|\left|\nabla \bar{u}^{n}\right|\right)\left|\bar{h}^{n+1}\right| .
\end{aligned}
$$

Then the second integral on the right-hand side of (3.23) can be controlled as

$$
\int \bar{u}^{n+1} \cdot \nabla p^{n+1} \bar{h}^{n+1} \leq C \int\left|\bar{u}^{n+1}\right|\left|\nabla \rho^{n+1}\right|\left|\bar{h}^{n+1}\right| .
$$

Next, applying integration by parts to the third integral on the right-hand side of (3.23), we easily get

$$
\int u^{n} \cdot \nabla \bar{p}^{n+1} \bar{h}^{n+1} \leq C \int\left|\nabla u^{n}\right|\left|\bar{\rho}^{n+1}\right|\left|\bar{h}^{n+1}\right|+C \int\left|u^{n}\right|\left|\bar{\rho}^{n+1}\right|\left|\nabla \bar{h}^{n+1}\right| .
$$

Consequently, combining (3.23)-(3.27) and using the Hölder, Sobolev, and Young inequalities and (3.2), one obtains

$$
\begin{aligned}
M_{4} \leq & \widetilde{C}\left(1+\eta^{-1}\right)\left(\left\|\bar{\rho}^{n+1}\right\|_{L^{2}}^{2}+\left\|\sqrt{\rho^{n+1}} \bar{h}^{n+1}\right\|_{L^{2}}^{2}\right) \\
& +\frac{1}{4}\left\|\bar{u}^{n+1}\right\|_{H^{1}}^{2}+\frac{1}{20}\left\|\nabla \bar{h}^{n+1}\right\|_{L^{2}}^{2}+\widetilde{C} \eta\left\|\bar{u}^{n}\right\|_{H^{1}}^{2} .
\end{aligned}
$$

Finally, we evaluate $M_{5}$. Direct calculation yields

$$
\begin{aligned}
M_{5} \leq & C \int\left(\left|\nabla u^{n}\right|+\left|\nabla u^{n-1}\right|\right)\left|\nabla \bar{u}^{n}\right|\left|\bar{h}^{n+1}\right|+C \int\left|\bar{\rho}^{n+1}\right|\left|\nabla \rho^{n+1}\right|^{2}\left|\bar{h}^{n+1}\right| \\
& +\int \frac{\mu_{t}}{\left(\rho^{n}\right)^{2}} \partial_{j} \bar{p}^{n+1} \partial_{j} \rho^{n+1} \cdot \bar{h}^{n+1}+\int \frac{\mu_{t}}{\left(\rho^{n}\right)^{2}} \partial_{j} p^{n} \partial_{j} \bar{\rho}^{n+1} \cdot \bar{h}^{n+1}
\end{aligned}
$$




$$
\begin{aligned}
& \leq C \int\left(\left|\nabla u^{n}\right|+\left|\nabla u^{n-1}\right|\right)\left|\nabla \bar{u}^{n}\right|\left|\bar{h}^{n+1}\right|+\left.C \int\left|\bar{\rho}^{n+1}\right|\left|\nabla \rho^{n+1}\right|\right|^{2}\left|\bar{h}^{n+1}\right| \\
& +C \int\left|\nabla \rho^{n}\right|\left|\nabla \rho^{n+1}\right|\left|\bar{\rho}^{n+1}\right|\left|\bar{h}^{n+1}\right|+C \int\left|\nabla^{2} \rho^{n+1}\right|\left|\bar{\rho}^{n+1}\right|\left|\bar{h}^{n+1}\right| \\
& \quad+C \int\left|\nabla \rho^{n+1}\right|\left|\bar{\rho}^{n+1}\right|\left|\nabla \bar{h}^{n+1}\right|+C \int\left|\nabla \rho^{n}\right|^{2}\left|\bar{\rho}^{n+1}\right|\left|\bar{h}^{n+1}\right| \\
& +C \int\left|\nabla^{2} \rho^{n}\right|\left|\bar{\rho}^{n+1}\right|\left|\bar{h}^{n+1}\right|+C \int\left|\nabla \rho^{n}\right|\left|\bar{\rho}^{n+1}\right|\left|\nabla \bar{h}^{n+1}\right| .
\end{aligned}
$$

Then, applying a similar method to deriving (3.28), one deduces

$$
M_{5} \leq \widetilde{C}\left(1+\eta^{-1}\right)\left(\left\|\bar{\rho}^{n+1}\right\|_{L^{2}}^{2}+\left\|\sqrt{\rho^{n+1}} \bar{h}^{n+1}\right\|_{L^{2}}^{2}\right)+\eta\left\|\bar{u}^{n}\right\|_{H^{1}}^{2}+\frac{1}{20}\left\|\nabla \bar{h}^{n+1}\right\|_{L^{2}}^{2}
$$

Consequently, inserting (3.20)-(3.22), (3.28), and (3.30) into (3.19), one gets

$$
\begin{aligned}
\frac{\mathrm{d}}{\mathrm{d} t}\left\|\sqrt{\rho^{n+1}} \bar{h}^{n+1}\right\|_{L^{2}}^{2}+\left\|\bar{h}^{n+1}\right\|_{H^{1}}^{2} \leq & \widetilde{C}\left(1+\eta^{-1}+\left\|h_{t}^{n}\right\|_{L^{3}}^{2}\right)\left(\left\|\bar{\rho}^{n+1}\right\|_{L^{2}}^{2}+\left\|\sqrt{\rho^{n+1}} \bar{h}^{n+1}\right\|_{L^{2}}^{2}\right) \\
& +\frac{1}{4}\left\|\bar{u}^{n+1}\right\|_{H^{1}}^{2}+\tilde{C} \eta\left\|\bar{u}^{n}\right\|_{H^{1}}^{2}
\end{aligned}
$$

For the turbulent kinetic energy $k$, using a similar method of deriving (3.19), one easily deduces from equation (3.6) that

$$
\begin{aligned}
\frac{1}{2} & \frac{\mathrm{d}}{\mathrm{d} t}\left\|{\sqrt{\rho^{n+1}}}_{\bar{k}}^{n+1}\right\|_{L^{2}}^{2}+\left\|\nabla \bar{k}^{n+1}\right\|_{L^{2}}^{2} \\
= & -\int \bar{\rho}^{n+1} k_{t}^{n} \cdot \bar{k}^{n+1}-\int \bar{\rho}^{n+1} u^{n} \cdot \nabla k^{n} \cdot \bar{k}^{n+1} \\
& \quad-\int \rho^{n} \bar{u}^{n} \cdot \nabla k^{n} \cdot \bar{k}^{n+1}+\int\left(G_{n+1}^{\prime}-G_{n}^{\prime}\right) \cdot \bar{k}^{n+1}-\int\left(\rho^{n+1} \varepsilon^{n}-\rho^{n} \varepsilon^{n-1}\right) \cdot \bar{k}^{n+1} \\
= & \sum_{i=1}^{5} N_{i} .
\end{aligned}
$$

We first evaluate $N_{4}$. Using the inserting items technique, one easily gets

$$
\begin{aligned}
N_{4} \leq & C \int\left(\left|\nabla u^{n}\right|+\left|\nabla u^{n-1}\right|\right)\left|\nabla \bar{u}^{n}\right|\left|\bar{k}^{n+1}\right| \\
& +C \int\left(\left|\nabla \bar{u}^{n}\right|+\left|\nabla u^{n-1}\right|\left|\bar{\rho}^{n+1}\right|+\left|\nabla u^{n-1}\right|\left|\bar{k}^{n}\right|\right)\left|\bar{k}^{n+1}\right| .
\end{aligned}
$$

Using the Hölder, Sobolev, and Young inequalities and (3.2), we have

$$
N_{4} \leq \widetilde{C}\left(1+\eta^{-1}\right)\left(\left\|\bar{\rho}^{n+1}\right\|_{L^{2}}^{2}+\left\|{\sqrt{\rho^{n+1}}}^{n+1}\right\|_{L^{2}}^{2}\right)+\widetilde{C} \eta\left\|\bar{k}^{n}\right\|_{H^{1}}^{2}+\widetilde{C} \eta\left\|\bar{u}^{n}\right\|_{H^{1}}^{2}
$$

Second, we estimate $N_{5}$. Using a similar method to deriving (3.33) and (3.34), we have

$$
\begin{aligned}
& N_{5}=\int\left(\bar{\rho}^{n+1} \varepsilon^{n}+\rho^{n} \bar{\varepsilon}^{n}\right) \cdot \bar{k}^{n+1} \leq C\left(\left\|\bar{\rho}^{n+1}\right\|_{L^{2}}\left\|\varepsilon^{n}\right\|_{L^{\infty}}+\left\|\bar{\varepsilon}^{n}\right\|_{L^{6}}\left\|\rho^{n}\right\|_{L^{3}}\right)\left\|{\sqrt{\rho^{n+1}}}_{\bar{k}^{n+1}}\right\|_{L^{2}} \\
& \leq \widetilde{C}\left(1+\eta^{-1}\right)\left(\left\|\sqrt{\rho^{n+1}} \bar{k}^{n+1}\right\|_{L^{2}}^{2}+\left\|\bar{\rho}^{n+1}\right\|_{L^{2}}^{2}\right)+\widetilde{C} \eta\left\|\bar{\varepsilon}^{n}\right\|_{H^{1}}^{2} .
\end{aligned}
$$


Next, using a similar method to deriving the estimates of (3.13), (3.14), and (3.15), respectively, one easily gets

$$
\begin{aligned}
& N_{1} \leq \widetilde{C}\left\|k_{t}^{n}\right\|_{L^{3}}^{2}\left\|\bar{\rho}^{n+1}\right\|_{L^{2}}^{2}+\widetilde{C}\left\|\sqrt{\rho^{n+1}} \bar{k}^{n+1}\right\|_{L^{2}}+\frac{1}{8}\left\|\nabla \bar{k}^{n+1}\right\|_{L^{2}}^{2}, \\
& N_{2} \leq \widetilde{C}\left\|\bar{\rho}^{n+1}\right\|_{L^{2}}^{2}+\widetilde{C}\left\|\sqrt{\rho^{n+1}} \bar{k}^{n+1}\right\|_{L^{2}}^{2}+\frac{1}{8}\left\|\nabla \bar{k}^{n+1}\right\|_{L^{2}}^{2}, \\
& N_{3} \leq \widetilde{C} \eta^{-1}\left\|\sqrt{\rho^{n+1}} \bar{k}^{n+1}\right\|_{L^{2}}^{2}+\eta\left\|\bar{u}^{n}\right\|_{H^{1}}^{2} .
\end{aligned}
$$

Consequently, inserting (3.34)-(3.38) to (3.32), one deduces

$$
\begin{gathered}
\frac{\mathrm{d}}{\mathrm{d} t}\left\|\sqrt{\rho^{n+1}} \bar{k}^{n+1}\right\|_{L^{2}}^{2}+\left\|\bar{k}^{n+1}\right\|_{H^{1}}^{2} \leq \\
\widetilde{C}\left(1+\eta^{-1}+\left\|k_{t}^{n}\right\|_{L^{3}}^{2}\right)\left(\left\|\sqrt{\rho^{n+1}} \bar{k}^{n+1}\right\|_{L^{2}}^{2}+\left\|\bar{\rho}^{n+1}\right\|_{L^{2}}^{2}\right) \\
+\widetilde{C} \eta\left(\left\|\bar{k}^{n}\right\|_{H^{1}}^{2}+\left\|\bar{u}^{n}\right\|_{H^{1}}^{2}+\left\|\bar{\varepsilon}^{n}\right\|_{H^{1}}^{2}\right) .
\end{gathered}
$$

Next, multiplying both sides of (3.7) by $\bar{\varepsilon}^{n+1}$ and integrating the result over $\Omega$, one gets

$$
\begin{aligned}
\frac{1}{2} & \frac{\mathrm{d}}{\mathrm{d} t}\left\|\sqrt{\rho^{n+1}} \bar{\varepsilon}^{n+1}\right\|_{L^{2}}^{2}+\left\|\nabla \bar{\varepsilon}^{n+1}\right\|_{L^{2}}^{2} \\
= & -\int \bar{\rho}^{n+1} \varepsilon_{t}^{n} \cdot \bar{\varepsilon}^{n+1}-\int \bar{\rho}^{n+1} u^{n} \cdot \nabla \varepsilon^{n} \cdot \bar{\varepsilon}^{n+1} \\
& -\int \rho^{n} \bar{u}^{n} \cdot \nabla \varepsilon^{n} \cdot \bar{\varepsilon}^{n+1}+C_{1} \int\left(\frac{G_{n+1}^{\prime} \varepsilon^{n}}{k^{n}}-\frac{G_{n}^{\prime} \varepsilon^{n-1}}{k^{n-1}}\right) \cdot \bar{\varepsilon}^{n+1} \\
& \quad-C_{2} \int\left[\frac{\rho^{n+1}\left(\varepsilon^{n}\right)^{2}}{k^{n}}-\frac{\rho^{n}\left(\varepsilon^{n-1}\right)^{2}}{k^{n-1}}\right] \cdot \bar{\varepsilon}^{n+1} \\
= & \sum_{i=1}^{5} Q_{i} .
\end{aligned}
$$

Using an argument similar to that used in deriving (3.13), (3.14), and (3.15), respectively, we obtain

$$
\begin{aligned}
& Q_{1} \leq \widetilde{C}\left\|\varepsilon_{t}^{n}\right\|_{L^{3}}^{2}\left\|\bar{\rho}^{n+1}\right\|_{L^{2}}^{2}+\widetilde{C}\left\|\sqrt{\rho^{n+1}} \bar{\varepsilon}^{n+1}\right\|_{L^{2}}^{2}+\frac{1}{8}\left\|\nabla \bar{\varepsilon}^{n+1}\right\|_{L^{2}}^{2}, \\
& Q_{2} \leq \widetilde{C}\left\|\bar{\rho}^{n+1}\right\|_{L^{2}}^{2}+\widetilde{C}\left\|\sqrt{\rho^{n+1}} \bar{\varepsilon}^{n+1}\right\|_{L^{2}}^{2}+\frac{1}{8}\left\|\nabla \bar{\varepsilon}^{n+1}\right\|_{L^{2}}^{2}, \\
& Q_{3} \leq \widetilde{C} \eta^{-1}\left\|\sqrt{\rho^{n+1}} \bar{\varepsilon}^{n+1}\right\|_{L^{2}}^{2}+\widetilde{C} \eta\left\|\bar{u}^{n}\right\|_{H^{1}}^{2} .
\end{aligned}
$$

Next, direct calculation leads to

$$
\begin{aligned}
Q_{4} \leq & C \int\left(\left|\nabla \bar{u}^{n}\right|\left|\nabla u^{n}\right|+\left|\nabla \bar{u}^{n}\right|\left|\nabla u^{n-1}\right|\right)\left|\varepsilon^{n}\right|\left|\bar{\varepsilon}^{n+1}\right| \\
& +C \int\left(\left|\bar{\varepsilon}^{n}\right|+\left|\varepsilon^{n-1}\right|\left|\bar{k}^{n}\right|\right)\left|\nabla u^{n-1}\right|^{2}\left|\bar{\varepsilon}^{n+1}\right| \\
& -\frac{2 C_{1}}{3} \delta_{i j} \int \frac{\left(\partial_{j} u_{i}^{n} \rho^{n+1} k^{n} \varepsilon^{n} k^{n-1}-\partial_{j} u_{i}^{n-1} \rho^{n} k^{n-1} \varepsilon^{n-1} k^{n}\right)}{k^{n} k^{n-1}} \cdot \bar{\varepsilon}^{n+1} \\
\leq & \int\left(\left|\nabla \bar{u}^{n}\right|\left|\nabla u^{n}\right|+\left|\nabla \bar{u}^{n}\right|\left|\nabla u^{n-1}\right|\right)\left|\varepsilon^{n}\right|\left|\bar{\varepsilon}^{n+1}\right|
\end{aligned}
$$




$$
\begin{aligned}
& +C \int\left(\left|\bar{\varepsilon}^{n}\right|+\left|\varepsilon^{n-1}\right|\left|\bar{k}^{n}\right|\right)\left|\nabla u^{n-1}\right|^{2}\left|\bar{\varepsilon}^{n+1}\right| \\
& +C \int\left(\left|\nabla \bar{u}^{n}\right|+\left|\nabla u^{n-1}\right|\left|\bar{\rho}^{n+1}\right|+\left|\nabla u^{n-1}\right|\left|\bar{k}^{n}\right|\right)\left|\varepsilon^{n}\right|\left|\bar{\varepsilon}^{n+1}\right| \\
& +C \int\left(\left|\bar{\varepsilon}^{n}\right|+\left|\varepsilon^{n-1}\right|\left|\bar{k}^{n}\right|\right)\left|\nabla u^{n-1}\right|\left|\bar{\varepsilon}^{n+1}\right| \\
& \leq \widetilde{C}\left(1+\eta^{-1}\right)\left(\left\|\sqrt{\rho^{n+1}} \bar{\varepsilon}^{n+1}\right\|_{L^{2}}^{2}+\left\|\bar{\rho}^{n+1}\right\|_{L^{2}}^{2}\right) \\
& +\widetilde{C} \eta\left(\left\|\bar{u}^{n}\right\|_{H^{1}}^{2}+\left\|\bar{k}^{n}\right\|_{H^{1}}^{2}+\left\|\bar{\varepsilon}^{n}\right\|_{H^{1}}^{2}\right)+\frac{1}{8}\left\|\nabla \bar{\varepsilon}^{n+1}\right\|_{L^{2}}^{2} .
\end{aligned}
$$

Finally, using a similar method of deriving the estimate of $Q_{4}$, one deduces

$$
Q_{5} \leq \widetilde{C}\left(1+\eta^{-1}\right)\left(\left\|\sqrt{\rho^{n+1}} \bar{\varepsilon}^{n+1}\right\|_{L^{2}}^{2}+\left\|\bar{\rho}^{n+1}\right\|_{L^{2}}^{2}\right)+\widetilde{C} \eta\left\|\nabla \bar{\varepsilon}^{n}\right\|_{L^{2}}^{2}+\frac{1}{8}\left\|\nabla \bar{\varepsilon}^{n+1}\right\|_{L^{2}}^{2} .
$$

Consequently, inserting (3.41)-(3.45) to (3.40), one derives

$$
\begin{aligned}
\frac{\mathrm{d}}{\mathrm{d} t} \| & \sqrt{\rho^{n+1}} \bar{\varepsilon}^{n+1}\left\|_{L^{2}}^{2}+\right\| \bar{\varepsilon}^{n+1} \|_{H^{1}}^{2} \\
\leq & \widetilde{C}\left(1+\eta^{-1}+\left\|\varepsilon_{t}^{n}\right\|_{L^{3}}^{2}\right)\left(\left\|\sqrt{\rho^{n+1}} \bar{\varepsilon}^{n+1}\right\|_{L^{2}}^{2}+\left\|\bar{\rho}^{n+1}\right\|_{L^{2}}^{2}\right) \\
& +\widetilde{C} \eta\left(\left\|\bar{k}^{n}\right\|_{H^{1}}^{2}+\left\|\bar{u}^{n}\right\|_{H^{1}}^{2}+\left\|\bar{\varepsilon}^{n}\right\|_{H^{1}}^{2}\right) .
\end{aligned}
$$

In the end, combining (3.11), (3.18), (3.31), (3.39), and (3.46), and setting $\varphi^{n+1}(t)=$ $\left\|\bar{\rho}^{n+1}\right\|_{L^{2}}^{2}+\left\|\sqrt{\rho^{n+1}} \bar{u}^{n+1}\right\|_{L^{2}}^{2}+\left\|\sqrt{\rho^{n+1}} \bar{h}^{n+1}\right\|_{L^{2}}^{2}+\left\|\sqrt{\rho^{n+1}} \bar{k}^{n+1}\right\|_{L^{2}}^{2}+\left\|\sqrt{\rho^{n+1}} \bar{\varepsilon}^{n+1}\right\|_{L^{2}}^{2}$, we get

$$
\begin{aligned}
& \frac{\mathrm{d}}{\mathrm{d} t} \varphi^{n+1}(t)+\left\|\bar{u}^{n+1}\right\|_{H^{1}}^{2}+\left\|\bar{h}^{n+1}\right\|_{H^{1}}^{2}+\left\|\bar{k}^{n+1}\right\|_{H^{1}}^{2}+\left\|\bar{\varepsilon}^{n+1}\right\|_{H^{1}}^{2} \\
& \leq \widetilde{C}\left(1+\eta^{-1}+\left\|u_{t}^{n}\right\|_{L^{3}}^{2}+\left\|h_{t}^{n}\right\|_{L^{3}}^{2}+\left\|k_{t}^{n}\right\|_{L^{3}}^{2}+\left\|\varepsilon_{t}^{n}\right\|_{L^{3}}^{2}\right) \varphi^{n+1}(t) \\
& \quad+\widetilde{C} \eta\left(\left\|\bar{u}^{n}\right\|_{H^{1}}^{2}+\left\|\bar{k}^{n}\right\|_{H^{1}}^{2}+\left\|\bar{\varepsilon}^{n}\right\|_{H^{1}}^{2}\right) .
\end{aligned}
$$

Setting $I_{\eta}^{n}(t)=\widetilde{C}\left(1+\eta^{-1}+\left\|u_{t}^{n}\right\|_{L^{3}}^{2}+\left\|h_{t}^{n}\right\|_{L^{3}}^{2}+\left\|k_{t}^{n}\right\|_{L^{3}}^{2}+\left\|\varepsilon_{t}^{n}\right\|_{L^{3}}^{2}\right)$ and applying the Gronwall inequality to (3.47) yield

$$
\varphi^{n+1}(t) \leq \widetilde{C} \eta\left[\exp \left(\int_{0}^{t} I_{\eta}^{n}(s) \mathrm{d} s\right)\right]\left(\int_{0}^{t}\left(\left\|\bar{u}^{n}\right\|_{H^{1}}^{2}+\left\|\bar{k}^{n}\right\|_{H^{1}}^{2}+\left\|\bar{\varepsilon}^{n}\right\|_{H^{1}}^{2}\right) \mathrm{d} s\right),
$$

where it should be noted that $\varphi^{n+1}(0)=0$.

Since

$$
\int_{0}^{t} I_{\eta}^{n}(s) \mathrm{d} s \leq \widetilde{C} t+\widetilde{C} \eta^{-1} t+\widetilde{C}
$$

setting $\widetilde{T} \leq \eta<1$, we have

$$
\int_{0}^{t} I_{\eta}^{n}(s) \mathrm{d} s \leq C \widetilde{C}
$$

for $t \leq \widetilde{T}$. 
By (3.48)-(3.50), integrating (3.47) from $[0, t]$, one derives

$$
\begin{aligned}
& \varphi^{n+1}(t)+\int_{0}^{t}\left(\left\|\bar{u}^{n+1}\right\|_{H^{1}}^{2}+\left\|\bar{h}^{n+1}\right\|_{H^{1}}^{2}+\left\|\bar{k}^{n+1}\right\|_{H^{1}}^{2}+\left\|\bar{\varepsilon}^{n+1}\right\|_{H^{1}}^{2}\right) \mathrm{d} s \\
& \quad \leq C \widetilde{C} \eta\left(\int_{0}^{t}\left(\left\|\bar{u}^{n}\right\|_{H^{1}}^{2}+\left\|\bar{k}^{n}\right\|_{H^{1}}^{2}+\left\|\bar{\varepsilon}^{n}\right\|_{H^{1}}^{2}\right) \mathrm{d} s\right)\left[\left(\int_{0}^{t} I_{\eta}^{n}(s) \mathrm{d} s\right) \exp \left(\int_{0}^{t} I_{\eta}^{n}(s) \mathrm{d} s\right)+1\right] \\
& \quad \leq C \eta \exp (\widetilde{C}) \int_{0}^{t}\left(\left\|\bar{u}^{n}\right\|_{H^{1}}^{2}+\left\|\bar{k}^{n}\right\|_{H^{1}}^{2}+\left\|\bar{\varepsilon}^{n}\right\|_{H^{1}}^{2}\right) \mathrm{d} s
\end{aligned}
$$

for $T^{*}:=\min \{T, \widetilde{T}\}$.

Therefore, we have

$$
\begin{aligned}
& \sum_{n=1}^{\infty} \sup _{0 \leq t \leq T} \varphi^{n+1}(t)+\sum_{n=1}^{\infty} \int_{0}^{t}\left(\left\|\bar{u}^{n+1}\right\|_{H^{1}}^{2}+\left\|\bar{h}^{n+1}\right\|_{H^{1}}^{2}+\left\|\bar{k}^{n+1}\right\|_{H^{1}}^{2}+\left\|\bar{\varepsilon}^{n+1}\right\|_{H^{1}}^{2}\right) \mathrm{d} s \\
& \quad \leq C \eta \exp (\widetilde{C}) \sum_{n=1}^{\infty} \int_{0}^{t}\left(\left\|\bar{u}^{n}\right\|_{H^{1}}^{2}+\left\|\bar{k}^{n}\right\|_{H^{1}}^{2}+\left\|\bar{\varepsilon}^{n}\right\|_{H^{1}}^{2}\right) \mathrm{d} s .
\end{aligned}
$$

Thus, choosing $\eta$ such that $C \eta \exp (\widetilde{C}) \leq \frac{1}{2}$, one deduces

$$
\begin{aligned}
& \sum_{n=1}^{\infty} \sup _{0 \leq t \leq T} \varphi^{n+1}(t)+\sum_{n=1}^{\infty} \int_{0}^{t}\left\|\bar{h}^{n+1}\right\|_{H^{1}}^{2} \mathrm{~d} s+\frac{1}{2} \sum_{n=1}^{\infty} \int_{0}^{t}\left(\left\|\bar{u}^{n+1}\right\|_{H^{1}}^{2}+\left\|\bar{k}^{n+1}\right\|_{H^{1}}^{2}+\left\|\bar{\varepsilon}^{n+1}\right\|_{H^{1}}^{2}\right) \mathrm{d} s \\
& \quad \leq C \widetilde{C}<\infty .
\end{aligned}
$$

Therefore, we conclude that the full sequence $\left(\rho^{n}, u^{n}, h^{n}, k^{n}, \varepsilon^{n}\right)$ converges to a limit $(\rho, u, h$, $k, \varepsilon)$ in the following strong sense: $\rho^{n} \rightarrow \rho$ in $L^{\infty}\left(0, T ; L^{2}(\Omega)\right) ;\left(u^{n}, h^{n}, k^{n}, \varepsilon^{n}\right) \rightarrow(u, h, k, \varepsilon)$ in $L^{2}\left(0, T ; H^{1}(\Omega)\right)$. It is easy to prove that the limit $(\rho, u, h, k, \varepsilon)$ is a weak solution to the original nonlinear problem. Furthermore, it follows from (3.2) that $(\rho, u, h, k, \varepsilon)$ satisfies the following regularity estimates:

$$
\begin{aligned}
& \sup _{0 \leq t \leq T^{*}}\left(\|\rho\|_{H^{3}}+\left\|\rho_{t}\right\|_{H^{1}}\right)+\sup _{0 \leq t \leq T^{*}}\left(\|u\|_{H^{3}}+\|k\|_{H^{2}}+\|\varepsilon\|_{H^{2}}+\|h\|_{H^{2}}\right) \\
& \quad+\sup _{0 \leq t \leq T^{*}}\left(\left\|\sqrt{\rho} u_{t}\right\|_{L^{2}}+\left\|\sqrt{\rho} h_{t}\right\|_{L^{2}}+\left\|\sqrt{\rho} k_{t}\right\|_{L^{2}}+\left\|\sqrt{\rho} \varepsilon_{t}\right\|_{L^{2}}\right) \\
& \quad+\int_{0}^{T^{*}}\left(\left\|u_{t}\right\|_{H^{1}}^{2}+\left\|h_{t}\right\|_{H^{1}}^{2}+\left\|k_{t}\right\|_{H^{1}}^{2}+\left\|\varepsilon_{t}\right\|_{H^{1}}^{2}+\|u\|_{H^{4}}^{2}+\|k\|_{H^{3}}^{2}\right) \leq \widetilde{C}<\infty .
\end{aligned}
$$

This proves the existence of a strong solution. Then we can easily prove the time continuity of the solution $(\rho, u, h, k, \varepsilon)$ by adapting the arguments in $[9,13]$. Finally, we prove the uniqueness. In fact, assume $\left(\rho_{1}, u_{1}, h_{1}, k_{1}, \varepsilon_{1}\right)$ and $\left(\rho_{2}, u_{2}, h_{2}, k_{2}, \varepsilon_{2}\right)$ be two strong solutions to the problem (1.1)-(1.10) with the regularity (3.1). Let $(\bar{\rho}, \bar{u}, \bar{h}, \bar{k}, \bar{\varepsilon})=\left(\rho_{1}-\rho_{2}, u_{1}-u_{2}, h_{1}-\right.$ $\left.h_{2}, k_{1}-k_{2}, \varepsilon_{1}-\varepsilon_{2}\right)$. Then following the same argument as in the derivations of (3.11), (3.18), (3.31), (3.39), and (3.46), we can prove that

$$
\begin{aligned}
& \frac{\mathrm{d}}{\mathrm{d} t}\left(\|\bar{\rho}\|_{L^{2}}^{2}+\left\|\sqrt{\rho_{1}} \bar{u}\right\|_{L^{2}}^{2}+\left\|\sqrt{\rho_{1}} \bar{h}\right\|_{L^{2}}^{2}+\left\|\sqrt{\rho_{1}} \bar{k}\right\|_{L^{2}}^{2}+\left\|\sqrt{\rho_{1}} \bar{\varepsilon}\right\|_{L^{2}}^{2}\right) \\
& \quad \leq R(t)\left(\|\bar{\rho}\|_{L^{2}}^{2}+\left\|\sqrt{\rho_{1}} \bar{u}\right\|_{L^{2}}^{2}+\left\|\sqrt{\rho_{1}} \bar{h}\right\|_{L^{2}}^{2}+\left\|\sqrt{\rho_{1}} \bar{k}\right\|_{L^{2}}^{2}+\left\|\sqrt{\rho_{1}} \bar{\varepsilon}\right\|_{L^{2}}^{2}\right)
\end{aligned}
$$


for some $R(t) \in L^{1}\left(0, T^{*}\right)$. Thus, by the Gronwall inequality, we conclude that $(\bar{\rho}, \bar{u}, \bar{h}, \bar{k}, \bar{\varepsilon})=$ $(0,0,0,0,0)$ in $\left(0, T^{*}\right) \times \Omega$. This completes the proof of Theorem 3.1 .

\section{Competing interests}

The authors declare that they have no competing interests.

\section{Authors' contributions}

The authors contributed equally in this article. They read and approved the final manuscript.

\section{Acknowledgement}

The research of BY was partially supported by the National Natural Science Foundation of China (No. 11471103).

Received: 12 November 2015 Accepted: 13 January 2016 Published online: 29 January 2016

\section{References}

1. Bian, DF, Guo, BL: Global existence of smooth solutions to the $k-\varepsilon$ model equations for turbulent flows. Commun. Math. Sci. 12, 707-721 (2014)

2. Launder, BE, Spalding, DB: Mathematical Models of Turbulence. Academic Press, New York (1972)

3. Lions, PL: Mathematical Topics in Fluid Mechanics, vol. 1. Oxford University Press, New York (1996)

4. Lions, PL: Mathematical Topics in Fluid Mechanics, vol. 2. Oxford University Press, New York (1998)

5. Feireisl, E: Dynamics of Viscous Compressible Fluids. Oxford University Press, Oxford (2004)

6. Feireisl, E: On the motion of a viscous, compressible, and heat conducting fluid. Indiana Univ. Math. J. 53, 1705-1738 (2004)

7. DiPerna, RJ, Lions, PL: Ordinary differential equations, transport theory and Sobolev spaces. Invent. Math. 98, 511-547 (1989)

8. Hoff, D: Strong convergence to global solutions for multidimensional flows of compressible, viscous fluids with polytropic equations of state and discontinuous initial data. Arch. Ration. Mech. Anal. 132, 1-14 (1995)

9. Cho, YG, Choe, HJ, Kim, HS: Unique solvability of the initial boundary value problems for compressible viscous fluid. J. Math. Pures Appl. 83, 243-275 (2004)

10. Cho, YG, Kim, HS: On classical solutions of the compressible Navier-Stokes equations with nonnegative initial densities. Manuscr. Math. 120, 91-129 (2006)

11. Choe, HJ, Kim, HS: Strong solutions of the Navier-Stokes equations for isentropic compressible fluids. J. Differ. Equ. 190, 504-523 (2003)

12. Salvi, R, Straskraba, I: Global existence for viscous compressible fluids and their behavior as $t \rightarrow \infty$. J. Fac. Sci., Univ. Tokyo, Sect. 1A, Math. 40, 17-51 (1993)

13. Choe, HJ, Kim, HS: Existence results for viscous polytropic fluids with vacuum. J. Differ. Equ. 228, $377-441$ (2006)

14. Xin, ZP: Blow-up of smooth solutions to the compressible Navier-Stokes equations with compact density. Commun. Pure Appl. Math. 51, 229-240 (1998)

15. Hu, XP, Wang, DH: Global solutions to the three-dimensional full compressible magnetohydrodynamic flows. Commun. Math. Phys. 283, 255-284 (2008)

16. $\mathrm{Hu}, \mathrm{XP}, \mathrm{Wang}, \mathrm{DH}$ : Compactness of weak solutions to the three-dimensional compressible magnetohydrodynamic equations. J. Differ. Equ. 245, 2176-2198 (2008)

17. Hu, XP, Wang, DH: Global existence and large-time behavior of solutions to the three dimensional equations of compressible magnetohydrodynamic flows. Arch. Ration. Mech. Anal. 197, 203-238 (2010)

18. Rozanova, O: Blow-up of smooth solutions to the barotropic compressible magnetohydrodynamic equations with finite mass and energy. In: Hyperbolic Problems: Theory, Numerics and Applications, pp. $911-917$ (2009)

19. Fan, JS, Yu, WH: Strong solution to the compressible magnetohydrodynamic equations with vacuum. Nonlinear Anal., Real World Appl. 10, 392-409 (2009)

\section{Submit your manuscript to a SpringerOpen ${ }^{\circ}$ journal and benefit from:}

- Convenient online submission

Rigorous peer review

- Immediate publication on acceptance

Open access: articles freely available online

- High visibility within the field

- Retaining the copyright to your article 